\title{
Zur Kenntnis \\ der Metalle der seltenen Erden
}

\author{
Von der \\ Technischen Hochschule der Freien Stadt Danzig \\ zur Erlangung der Würde eines Doktor-Ingenieurs \\ genehmigte \\ Dissertation
}

\author{
Vorgelegt von \\ Dipl.=Ing. Heinrich Bommer \\ aus Röhrigshof (Werra)
}

Referent: Prof. Dr. W. Klemm

Korreferent: Prof. Dr. H. Albers

Tag der Promotion: 3. April 1937

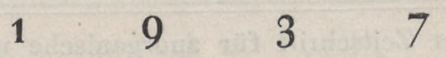

\begin{tabular}{llllllllllllllllllll}
\hline & e & o & p & o & l & d & $V$ & o & s & s & i & n & $L$ & e & i & p & z & i & g
\end{tabular} 


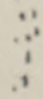

Sonderabdruck aus der Zeitschrift für anorganische und allgemeine Chemie Band 231 (1937), Heft 1-2

Printed in Germany

$$
\frac{B-k a \text { GPG }}{D / G-226 / 57}
$$



Von den Metallen der seltenen Erden sind bisher nur die bereits in der klassischen Untersuchung von Muthmann und Weiss ${ }^{1}$ ) dargestellten ersten Glieder Lanthan, Cer, Praseodym und Neodym gut bekannt. Außerdem ist in neuerer Zeit das Gadolinium dargestellt und magnetisch untersucht worden ${ }^{2}$ ). Weniger zuverlässig erscheinen die bisherigen Angaben über metallisches Samarium, das von MutHMANN und Weiss nur in unreiner Form erhalten wurde. Von den Yttererden ist außer Gd noch das Erbium dargestellt worden; jedoch sind die für dieses Element angegebenen physikalischen Daten sehr eigenartig: die von J. C. McLennan und R. J. Monkman ${ }^{3}$ ) bestimmte Gitterkonstante ist ungewöhnlich groß, die von M. OwEN ${ }^{4}$ ) an einem allerdings sehr unreinen Präparat gemessene magnetische Suszeptibilität ist außerordentlich klein.

Es war das Ziel der vorliegenden Untersuchung, möglichst die Metalle aller seltenen Erden herzustellen. Hierfür mußte eine Methode angewendet werden, die es gestattet, auch mit sehr geringen Substanzmengen die Metalle in einer Form zu erhalten, die die Ermittlung besonders wichtiger Eigenschaften ermöglicht. Gewählt wurde das alte WöHLER'sche Verfahren, die wasserfreien Chloride mit Alkalimetall $\mathrm{zu}$ reduzieren. (Über die im einzelnen angewandte $\mathrm{Me}$ thodik vgl. Teil A.) Man erhielt zwar dabei das Metall nicht in kompakter Form, sondern nur ein pulverförmiges Gemisch des Erdmetalls mit dem betreffenden Alkalichlorid. An einem solchen Gemisch lassen sich aber bereits die Kristallstruktur des Metalls und damit seine Dichte sowie die magnetischen Eigenschaften untersuchen. Das beigemengte Alkalichlorid dient bei den Pulveraufnahmen gleichzeitig

1) W. Muthmann u. L. Weiss, Lieb. Ann. 320 (1901), 231.

2) F. Trombe, Bull. Soc. chim. France [5] 2 (1935), 660; G. Urbain, P. Weiss u. F. Trombe, Compt. rend. 200 (1935), 2132.

3) J. C. Mo Lennan u. R. J. Monkman, Trans. Roy. Soc. Canada (3) Sect. III, 23 (1929), 255.

4) M. Owwe, Ann. Phys. 37 (1912), 657. 
als Eichsubstanz, bei der magnetischen Messung als ein bei dem zu erwartenden hohen Magnetismus der seltenen Erdmetalle erwünschtes Verdünnungsmittel.

Versuche ähnlicher Art sind bereits von E. Zintu und S. NeuMAYER ${ }^{1}$ ) durchgeführt worden. ZINTL reduzierte $\mathrm{CeCl}_{3}$ mit Na- und $\mathrm{K}-\mathrm{Dampf}$. Aus den erhaltenen Röntgendiagrammen und Versuchen über das. Verhalten von Cer-Mischmetall gegenüber Kalium-Dampf kam Zintu jedoch zu dem Schluß, daß die Erdmetalle Alkalimetall aufnehmen, zum Teil unter Gitteraufweitung. Wir führten unsere Versuche mit flüssigem Alkalimetall durch und benutzten neben Kaliumauch Caesium- und Rubidiummetall als Reduktionsmittel, da wir glaubten, daß diese sehr weiträumigen Metalle sich nicht so leicht im Erdmetall auflösen würden. Die Versuche an den bereits in metallischer Form bekannten seltenen Erden, über die Einzelheiten in den späteren Abschnitten mitgeteilt werden, ergaben folgendes:

1. Die Analysen der erhaltenen Gemische entsprachen, wie Tabelle 1 für einige herausgegriffene Beispiele zeigt, recht gut den Werten für Produkte der allgemeinen Zusammensetzung: Erdmetall +3 Alkalichlorid.

Tabelle 1

\begin{tabular}{|c|c|c|c|c|c|c|c|}
\hline \multirow{2}{*}{ Präparat } & \multicolumn{3}{|c|}{ Berechnet } & \multicolumn{4}{|c|}{ Gefunden } \\
\hline & selt. Erde & Chlor & Kalium & selt. Erde & Chlor & Kalium & Summe \\
\hline $\mathrm{La}+3 \mathrm{KCl}$ & 38,30 & 29,35 & 32,35 & 38,1 & 29,4 & 32,4 & 99,9 \\
\hline $\mathrm{Pr}+3 \mathrm{KCl}$ & 38,65 & 29,18 & 32,17 & 38,9 & 29,3 & 31,9 & 100,1 \\
\hline $\mathrm{Nd}+3 \mathrm{KCl}$ & 39,21 & 28,91 & 31,88 & 39,3 & 28,5 & 31,8 & 99,6 \\
\hline
\end{tabular}

2. Beim La, Ce, Pr und Nd erhielt man unabhängig von der Art des verwendeten Alkalimetalls $(\mathrm{K}, \mathrm{Rb}, \mathrm{Cs})$ neben den Linien der Alkalichloride stets die gleichen Röntgeninterferenzen.

3. Die aus diesen Diagrammen ermittelten Strukturen und Gitterabstände (vgl. B 2) entsprachen, wie die Tabelle 5 S. 149 zeigt, genau den von anderer Seite an kompaktem Material gefundenen Werten.

4. Als wir verschiedene Alkalimetalle im geschmolzenen Zustande auf kompaktes Lanthan einwirken ließen, zeigten die Reaktionsprodukte keinerlei Verschiebung der Röntgeninterferenzen gegenüber dem Ausgangsmaterial.

5. Schließlich stellten wir bei der magnetischen Untersuchung (näheres vgl. S.161 ff.) fest, daß die Suszeptibilitäten unserer La-, Ce-, Pr-, Nd- und Gd-Präparate recht gut den besten Literaturwerten entsprachen.

1) E. ZinTL u. S. NeUMAYGR, Z. Elektrochem. 39 (1933), 85. 
Nach diesen Versuchen schienen uns die Grundlagen genügend gesichert, um auf die gleiche Art die Gitterkonstanten und das magnetische Verhalten der bisher noch nicht dargestellten Erdmetalle zu untersuchen. Der vorliegende Bericht bringt unter B 3 Daten über die Gitterstrukturen von Eu, Gd, Tb, Dy, Er, Tm, Yb und Cp. Für das ebenfalls untersuchte Sm können wir dagegen bisher sichere Angaben über die Struktur noch nicht machen (vgl. dazu S. 157).

In C schließlich werden die erhaltenen Ergebnisse über den Verlauf der Atomradien, Atomvolumina, Gitterstrukturen usw. besprochen. Dieser Verlauf der Atomvolumina zeigt eine Reihe von Besonderheiten, zu deren Aufklärung magnetische Untersuchungen der Präparate erwünscht erschienen. Hierüber wird in $\mathrm{D}$ berichtet.

\section{A. Ausgangsmaterial; Darstellung der Präparate; Analysen}

1. Ausgangsmaterial. Die Cer-Erden (La, Ce, $\mathrm{Pr}, \mathrm{Nd}$ ) waren Präparate der Auergesellschaft, die bereits bei früheren Untersuchungen benutzt worden waren und sich als genügend rein erwiesen hatten. Genauere Angaben über den Reinheitsgrad können wir hier nicht machen. Bei magnetischen Messungen an den Oxyden erhielten wir Werte, die mit den besten Literaturdaten übereinstimmten. Die benutzten Präparate von Samarium, Dysprosium und Erbium stammten von dem verstorbenen Freiherrn Auer von WelsBach. Genauere Angaben über die Reinheit können wir nur über das Samarium machen. Für dieses hatte eine vor einigen Jahren von Herrn Dr. Beuthe, Charlottenburg, durchgeführte röntgenspektroskopische Analyse einen Gehalt von 0,8\% Eu und 0,3\% Gd ergeben. Das Gadoliniumoxyd stammte von Herrn Prof. Dr. JANTSCH in Graz, der uns mitteilte, daß die Reinheit mindestens $99,5 \%$ beträgt; der Rest besteht aus Samarium und den ersten Gliedern der Yttererdenreihe. Europiumoxyd stellte Herr cand. chem. DöLu über das $\mathrm{EuSO}_{4}$ her, wobei man die Erfahrungen von A. BRUKL ${ }^{1}$ ) und W. KAPFENBERGER ${ }^{2}$ ) benutzte. Nach einer von Frl. E. Mehnert und Herrn Brauer im Institut von Herrn Prof. Dr. Toмascheк in Dresden durchgeführten röntgenspektroskopischen Untersuchung waren auch bei sehr langer Belichtungszeit Linien von Fremdbestandteilen nicht zu erkennen. Außerdem stellte uns Herr Tomaschen freundlicherweise $200 \mathrm{mg}$ reines $\mathrm{Eu}_{2} \mathrm{O}_{3}$ zur Verfügung. Röntgenspektroskopisch reines Ytter-

1) A. BrukL, Z. Angew. Chemie 49 (1936), 32.

$\left.{ }^{2}\right)$ W. Kapfenberger, Z. analyt. Chem. 105 (1936), 199. 
biumoxyd verdanken wir Herrn Generaldirektor Dr. Dr.-Ing. e.h. Feit, Berlin.

Reine Präparate von $\mathrm{Tb}$, Tm und $\mathrm{Cp}$ haben wir bisher nicht untersuchen können. Das von uns benutzte Cassiopeium, das wir Herrn Prof. Dr. Prandtr, München, verdanken, war nach Angabe von Herrn Prandtu zwar frei von Yb, aber noch Tm-haltig. Nach unseren magnetischen Messungen enthielt es ungefähr $4 \%$ Tm. Thuliumoxyd, das noch $4,7 \% \quad \mathrm{Yb}_{2} \mathrm{O}_{3}$ und $5,7 \% \mathrm{Cp}_{2} \mathrm{O}_{3}$ enthielt, überließ uns ebenfalls Herr Feiт. Seiner Liebenswürdigkeit verdanken wir auch ein Terbiumoxyd-Präparat, bei dem der Gehalt an Terbium ungefähr 85\% der insgesamt vorhandenen Erden betrug.

Kalium und Natrium reinigten wir durch wiederholtes Destillieren im Hochvakuum. Rubidium und Caesium wurden nach der von W. Biltz, F. Weibke und H. EGGErs ${ }^{1}$ ) gegebenen Vorschrift durch Erhitzen der Chloride mit Calciumspänen in einem gut evakuierten Gerät aus Jenaer Glas gewonnen und in kleine Vorratsröhrchen destilliert, die abgeschmolzen wurden.

2. Darstellung der Präparate. Die von uns durchgeführte präparative Darstellung der Erdmetalle unterschied sich, wie bereits bemerkt, von dem Vorgehen von E. Zintu und S. Neumayer dadurch, daß wir nicht Alkalimetalldampf auf die Chloride einwirken ließen, sondern das flüssige Alkalimetall. Wir stellten nämlich fest, daß die Reaktion mit Alkalimetalldampf in der Regel nicht zu vollständiger Reduktion des Erdchlorids führt. Außerdem erhält man bei dieser Methode oft stark zusammengebackene Produkte, die erst zerkleinert werden müssen, wodurch die Schärfe der Röntgeninterferenzen leidet. Im Gegensatz dazu kommt man bei der Reduktion mit flüssigem Alkalimetall, die oft schon unter $200^{\circ} \mathrm{C}$ beginnt, zu einem lockeren Pulver, das auch beim späteren Erhitzen auf $350-400^{\circ} \mathrm{C}$ nicht mehr zusammenbackt, so daß ein nachträgliches Pulverisieren nicht mehr nötig ist.

Im einzelnen gingen wir folgendermaßen vor: Wir arbeiteten mit wasserfreiem Erdchlorid, das nach O. HöNIGscHMID und H. HoLCH ${ }^{2}$ ) hergestellt wurde, wobei aber nicht ganz bis zum Schmelzen erhitzte. Dabei überzeugte man sich in jedem Falle, daß sich das Chlorid ohne jede Trübung im Wasser löste, also oxydfrei war. Das Chlorid wurde unter sorgfältigem Ausschluß von Luft und Feuchtigkeit an die

1) W. Biltz, F. Weibke u. H. EgGers, Z. anorg. u. allg. Chem. 219 (1934), 119.

2) O. Hönigschmid u. H. HoLCH, Z. anorg. u. allg. Chem. 165 (1927), 294 und 177 (1928), 94. 
Stelle $A$ eines mit Argon gefüllten Geräts aus Supremaxglas, wie es in Fig. 1 dargestellt ist, gebracht. Dann führte man in das Rohr $B$ ein mit frisch destilliertem Alkalimetall gefülltes Glasröhrchen ein, von dem ganz kurz vorher eine Spitze abgebrochen war. Darauf wurde rasch bei $C$ abgeschmolzen und durch $D$ hoch evakuiert. Durch leichtes Erwärmen der Metallprobe bei $B$ wurde diese zum Schmelzen gebracht, so daß sie durch die enge Kapillare $E$ nach $A$ floß. Eventuell vorhandene geringe Oxydmengen wurden dabei von der Kapillare

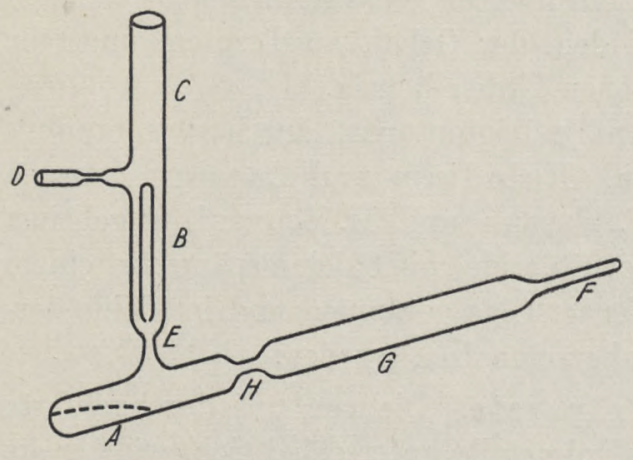

Fig. 1. Apparatur zur Darstellung der Erdmetalle zurückgehalten. Dann wurde bei $E$ abgeschmolzen und das Rohr $A F$ auf die Länge $A G$ im elektrischen Ofen erhitzt. Dieses Erhitzen wurde in der Regel zunächst bei niedriger Temperatur $\left(200-300^{\circ} \mathrm{C}\right)$ durchgeführt. Dabei fand die Reduktion statt und das überschüssige Alkalimetall destillierte langsam an die rechts von $G$ gelegenen Stellen des Rohres. Dann erhitzte man noch einige Stunden auf etwa $350^{\circ} \mathrm{C}^{1}$ ). Nach dem Erkalten wurde $F$ durch einen trockenen Gummischlauch mit der Vakuum-Argon-Apparatur verbunden und die Spitze $F$ unter Argon abgebrochen. Das Alkalimetall bei $G$ diente dabei gleichzeitig dazu, um Spuren von Wasserdampf, die aus dem Gummischlauch in das Gas kommen konnten, unschädlich zu machen. Jetzt konnte man bei $H$ ohne Gefahr für das Präparat absprengen und das bei $A$ befindliche Gemisch von Alkalichlorid und Erdmetall in eine mit Argon gefüllte Umfüllapparatur2) schütten. In dieser konnte das Präparat, wenn erforderlich, mit einem Glasstab gepulvert werden. Das Pulver wurde dann auf die Röhrchen für die magnetische Messung, die Markröhrchen für die Röntgenuntersuchung und Kölbchen für Analysenproben verteilt.

Schwierigkeiten zeigten sich, als man auf diese Weise Sa marium-, Europium- und Ytterbiumchlorid reduzieren wollte. In diesem Falle fand nur eine unvollständige Reduktion statt. Es hängt dies

1) Utber das etwas abweichende Vorgehen bei Sm, Eu und Yb vgl. S. 143.

$\left.{ }^{2}\right)$ Diese Umfüllapparatur ist in ihren wesentlichen Zügen von E. ZrNTL und A. HARDer, Z. phys. Chem. A 154 (1931), 92, beschrieben. 
offenbar damit zusammen, daß diese Elemente Dichloride bilden. Es wurden daher am $\mathrm{SmCl}_{3}$ systematische Versuche angestellt, bei denen man sowohl das Alkalimetall wie auch die Temperaturbedingungen variierte. Von den Alkalimetallen bewährte sich Natrium am wenigsten. Die Reduktion führte hier zu dunkelrotem $\mathrm{SmCl}_{2}$. Bessere Ergebnisse erhielt man mit den schwereren Alkalimetallen, insbesondere mit Kalium. Bezüglich der Temperatur stellte sich heraus, daß jede unnötige Temperatursteigerung zu vermeiden ist. Es findet nämlich, wie bereits Zintu und Neumayer festgestellt haben, bei höheren Temperaturen bei allen Elementen eine Rückbildung von Erdchlorid und Alkalimetall statt. Während aber diese rückläufige Reaktion bei der Mehrzahl der Erden unterhalb $400-500^{\circ} \mathrm{C}$ nicht nennenswert in Erscheinung tritt, wird sie bei den Dichloride bildenden Metallen schon bei $300^{\circ}$ merklich. Man durfte daher in diesen Fällen nur bis maximal $250^{\circ} \mathrm{C}$ erhitzen; dabei dauerte das Abdestillieren des überschüssigen Kaliums ungefähr 20 Stunden. Nach diesen Erfahrungen war es ohne Schwierigkeiten möglich, bei der Reduktion von $\mathrm{SmCl}_{3}, \mathrm{EuCl}_{3}$ und $\mathrm{YbCl}_{3}$ analytisch einwandfreie Präparate zu erhalten. Die Röntgendiagramme werden auch in diesen Fällen scharf. Beim Samarium ließen allerdings die Diagramme bisher keine Auswertung zu.

3. Analysen. Bei den ersten Analysen wurden alle drei Bestandteile (Chlor, Erdmetall und Alkalimetall) bestimmt. Vgl. Tabelle 1 S. 139. Bei späteren Proben begnügte man sich damit, Bestimmungen des Chlorid- und Erdgehalts durchzuführen, bei den letzten Versuchen wurde schließlich nur noch Chlor bestimmt. Eine Auswahl aus den Analysenergebnissen gibt Tabelle 2.

Tabelle 2

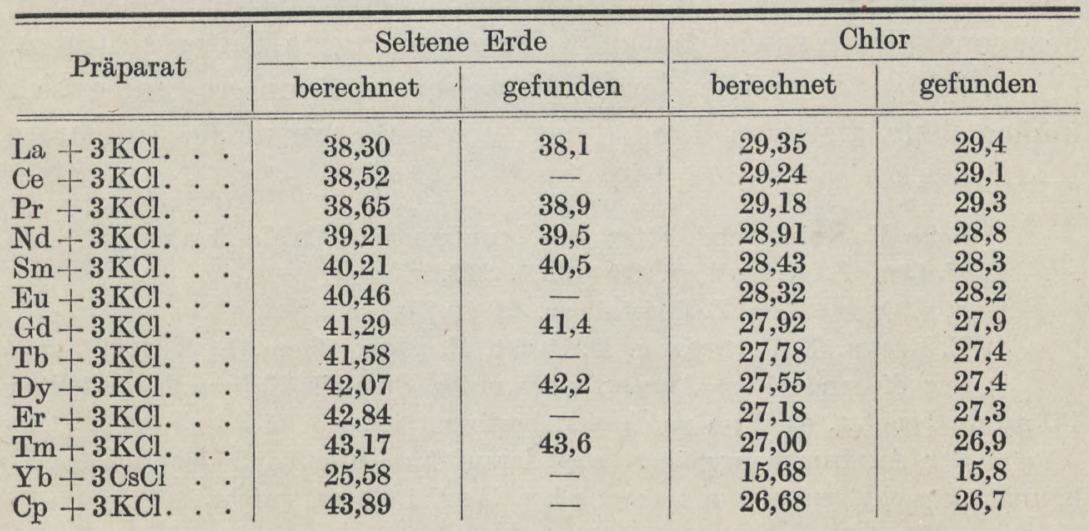


144 Zeitschrift für anorganische und allgemeine Chemie. Band 231. 1937

\section{B. Röntgenographischer Teil}

1. Allgemeines. Zur röntgenographischen Untersuchung wurden die Substanzproben unter Argon in Markröhrchen von $0,5-0,6 \mathrm{~mm}$ Dicke eingefüllt und diese abgeschmolzen. Belichtet wurde in der Regel mit Kupferstrahlung [Nickelfilter, Spannung $35 \mathrm{kV}, 10 \mathrm{~mA}$, $\left.\left.\mathrm{CuK} \alpha=1,539 \AA^{1}\right)\right]$. Die Belichtungsdauer betrug durchschnittlich 3 Stunden; der Kameradurchmesser war $57,3 \mathrm{~mm}$.

Die Diagramme enthielten neben den Linien des Erdmetalls noch die des Alkalichlorids. Obwohl in Molen gerechnet die Menge der Alkalichloride die dreifache war wie die des Erdmetalls, waren die Linien des Erdmetalls sehr deutlich zu erkennen. Ihre Intensität war in der Regel kaum schwächer, manchmal sogar stärker als die der Alkalichlorid-Linien. Die Ausmessung der Diagramme und die Abschätzung der Intensitäten machten daher keinerlei Schwierigkeiten.

Bei der Auswertung der Diagramme dienten die AlkalichloridLinien in bekannter Weise gleichzeitig zur Feinkorrektur des Kameraradius. Nach Anbringung der Korrektur für die Stäbchendicke waren die Abweichungen zwischen berechneten und gefundenen Alkalichlorid-Linien klein; sie betrugen kaum mehr als \pm 5 Minuten. Dabei benutzte man für $\mathrm{KCl}, \mathrm{RbCl}$ und $\mathrm{CsCl}$ folgende Gitterkonstanten: $\left.\left.\left.a_{\mathrm{KCl}}=6,277 \AA^{2}\right), \quad a_{\mathrm{RbCl}}=6,535 \AA^{3}\right), \quad a_{\mathrm{CsCl}}=4,113 \AA^{4}\right)$. Aus den korrigierten $\vartheta$-Werten ${ }^{5}$ ) der nicht dem Alkalichlorid zugehörigen Linien ermittelte man unter Benutzung der HuLL'schen Kurven zunächst den Gittertypus, ferner bei hexagonalen Gittern das ungefähre Achsenverhältnis und die Indizes der den einzelnen Linien zugehörigen Ebenen. Dies war durchweg ohne Schwierigkeiten möglich, da nur das kubisch-flächenzentrierte, das [angenähert $\left.{ }^{6}\right)$ ] hexagonal-dichte sowie das kubisch-raumzentrierte Gitter auftreten. Wir möchten besonders darauf hinweisen, daß unsere Filme sich immer dann frei von Fremdlinien erwiesen, wenn die Präparate

1) Beim Europium und Ytterbium benutzte $\operatorname{man}$ Co $K \alpha$-Strahlung.

2) H. Oтт, Z. Kristallogr. 63 (1926), 227.

3) S. v. Olshausen, Z. Kristallogr. 61 (1925), 463.

4) E. Broch, F. Oftedal u. A. Pabst, Z. phys. Chem. B, 3 (1929), 209.

${ }^{5}$ ) Für die endgültige Auswertung wurden die mit Kalium hergestellten Präparate benutzt, da $a_{\mathrm{KCl}}$ am besten bestimmt ist.

6) Der Ausdruck angenähert soll darauf hindeuten, daß das Achsenverhältnis $c / a$ nicht genau dem theoretischen Wert $1,63_{3}$ entspricht. 
einwandfrei waren $\left.{ }^{\mathbf{1}}\right)$. Unstimmigkeiten in der Analyse, d. h. unvollkommene Umsetzung, drückten sich in den Röntgendiagrammen stets durch flauere Filme und das Auftreten von Fremdlinien aus.

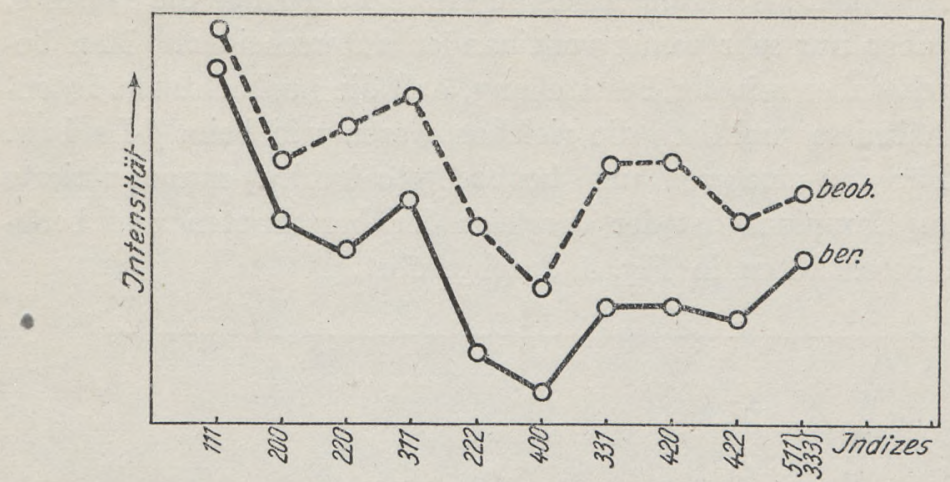

Fig. 2. Beob. und ber. Intensitäten für $\beta$-La (kub. flz.)

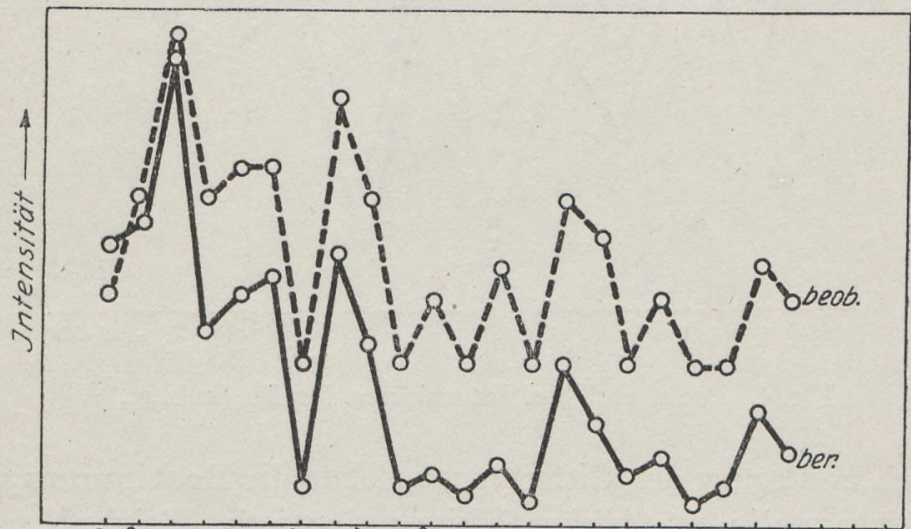

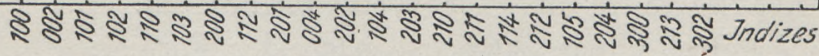

Fig. 3. Beob. und ber. Intensitäten für $\alpha$-Nd (hex. d.)

Intensitätsberechnungen wurden für $\beta$-La (kubisch-flächenzentriert) und $\alpha$-Nd (hexagonal-dicht) durchgeführt. Wie Fig. 2 und 3 zeigen, stimmen die nach der Gleichung:

$$
I \sim F \cdot \frac{1+\cos ^{2} 2 \vartheta}{\sin ^{2} \vartheta \cdot \cos \vartheta} \cdot S^{2}
$$

1) Nur beim $\beta$-Cer und $\beta$-Praseodym waren die beiden sehr starken Linien (101) und (103) der $\alpha$-Form als sehr schwache Linien zu erkennen. Wir vermuten, daß auch die von anderen Autoren beobachteten Fremdlinien zum Teil darauf zurückzuführen sind, daß man die $\alpha$ - oder $\beta$-Form nicht ganz rein in der Hand hatte. 
Zeitschrift für anorganische und allgemeine Chemie. Band 231. 1937

berechneten Intensitätsverhältnisse mit den beobachteten sehr gut überein $\left.{ }^{1}\right)$. Für die anderen Gitter dieser Typen war eine Berechnung nicht erforderlich; denn für die Berechnung der relativen Intensitäten erscheinen als Veränderliche in diesen Fällen nur die $\vartheta$-Werte, die sich aber nur sehr wenig voneinander unterscheiden. Man begnügte sich daher mit der Feststellung, daß die beobachteten Intensitätsverhältnisse praktisch die gleichen waren wie beim $\beta$-La bzw. $\alpha$-Nd. Für das eine von uns beobachtete kubisch-raumzentrierte Gitter des Europiums ist der Vergleich der berechneten und beobachteten Intensitäten in Fig. 4 durchgeführt.

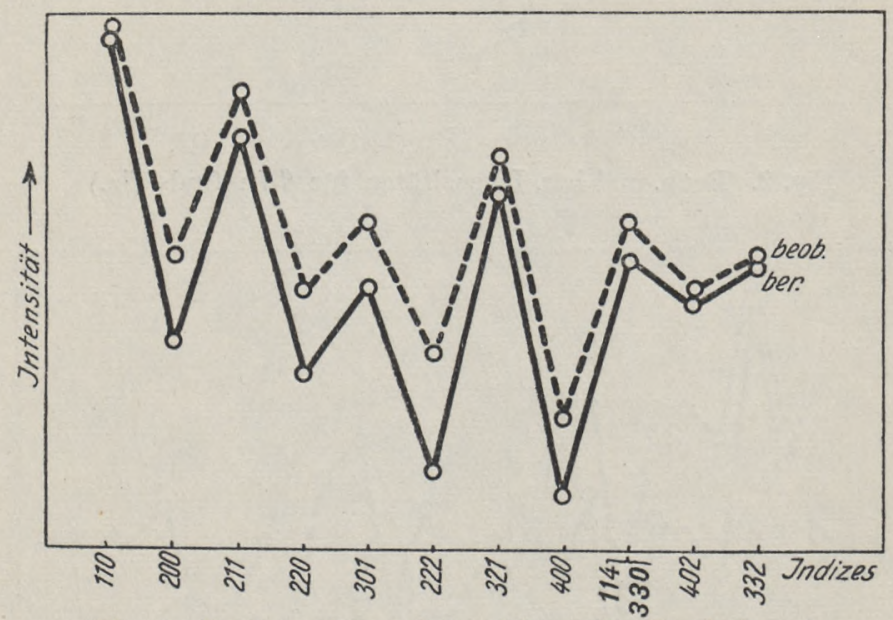

Fig. 4. Beob. und ber. Intensitäten für Eu (kub. rz.)

Nach Festlegung des Gittertypus erfolgte die Bestimmung der Elementarzelle in üblicher Weise rechnerisch. Bei kubischen Gittern ermittelte man zunächst nach der Formel:

$$
\sin ^{2} \vartheta=\frac{\lambda^{2}}{4 a^{2}} \cdot\left(h^{2}+k^{2}+l^{2}\right)
$$

die Gitterkonstante $a$. Aus dieser ergab sich dann beim kubischflächenzentrierten Gitter der Radius in Zwölferkoordination zu: $r^{[12]}=a / 4 \cdot \sqrt{2} \AA$. Das Atomvolumen beträgt:

$$
A V=\frac{a^{3} \cdot 10^{-24}}{4 \cdot 1,65 \cdot 10^{-24}} \mathrm{~cm}^{3}
$$

1) Die experimentelle Intensität der Linien, die beim $\alpha$-Nd mit KCl-Linien koinzidieren, wurde durch einen Vergleich mit andern Metallen erhalten, bei denen diese Koinzidenz nicht vorhanden ist. 
W. Klemm u. H. Bommer. Zur Kenntnis der Metalle der seltenen Erden 147

Beim kubisch-raumzentrierten Europium wird entsprechend der Radius in Achterkoordination $r^{[8]}=a / 4 \cdot \sqrt{3}=0,97 \cdot r^{[12]}$ und das Atomvolumen

$$
A V=\frac{a^{3} \cdot 10^{-24}}{2 \cdot 1,65 \cdot 10^{-24}} \mathrm{~cm}^{3}
$$

Bei den hexagonalen Gittern gilt:

$$
\sin ^{2} \vartheta=\frac{\lambda^{2}}{3 a^{2}}\left(h^{2}+h k+k^{2}\right)+\frac{\lambda^{2}}{4 c^{2}} \cdot l^{2} .
$$

Hier wurden, nachdem das ungefähre Achsenverhältnis $c / a$ aus den Hulz'schen Kurven ermittelt war, das genaue Verhältnis $c / a$ so festgelegt, daß man es solange variierte, bis die Werte von $\frac{\lambda^{2}}{3 a^{2}}=q$ und $\frac{\lambda^{2}}{4 c^{2}}=r$ untereinander möglichst geringe Abweichungen zeigten. Aus $a$ und $c$ folgt für den Atomradius: $r^{[12]}=\frac{a+e}{4}$, wobei $e=\sqrt{\frac{a^{2}}{3}+\frac{c^{2}}{4}}$ ist. Das Atomvolumen errechnet sich zu

$$
A V=\frac{a^{2} \cdot c \cdot \sqrt{3} \cdot 10^{-24}}{4 \cdot 1,65 \cdot 10^{-24}} \mathrm{~cm}^{3}
$$

Die Dichte ist in allen Fällen: $D_{R}=\frac{\text { Atomgewicht }}{\text { Atomvolumen }}$.

2. Erprobung der Meßmethode an La, Ce, Pr und Nd. Zur näheren Erläuterung des in B 1 beschriebenen Vorgehens sind unsere Versuchsergebnisse für einen $(\mathrm{Nd}+3 \mathrm{KCl})$-Film ausführlich in Tabelle 3 (S. 148) mitgeteilt. Tabelle 4 (S. 149) enthält in abgekürzter Form die beobachteten Werte für La, Ce und Pr, die bei unseren Versuchsbedingungen in der $\beta$-Form auftraten. Die angegebenen Zahlen stellen Mittelwerte aus untereinander sehr gut übereinstimmenden Messungen an folgenden Präparaten dar: Pulver von kompaktem Lanthanmetall, behandelt mit K-, Rb- und Cs-Metall; $\mathrm{LaCl}_{3}$ reduziert mit $\mathrm{K}$ und $\mathrm{Cs} ; \mathrm{CeCl}_{3}$ red. mit $\mathrm{K}$; $\mathrm{PrCl}_{3}$ red. mit $\mathrm{K}$ und $\mathrm{Cs} ; \mathrm{NdCl}_{3}$ red. mit $\mathrm{K}$.

Ein Vergleich der gefundenen Werte mit den Literaturdaten ist in Tabelle 5 (S. 149) gegeben. Die Genauigkeit unserer Messungen schätzen wir unter Berücksichtigung aller möglichen Fehlerquellen auf $\pm 0,003 \AA$. Die Übereinstimmung unserer Messungen mit den besten Literaturwerten bei La, Ce und Nd ist ausgezeichnet. Beim Praseodym war bisher nur die $\alpha$-Form bekannt. Der von uns aus der Gitterkonstanten für die $\beta$-Form ermittelte Atomradius für Pr stimmt recht gut mit dem aus der $\alpha$-Form abgeleiteteten überein. 
148 Zeitschrift für anorganische und allgemeine Chemie. Band 231. 1937

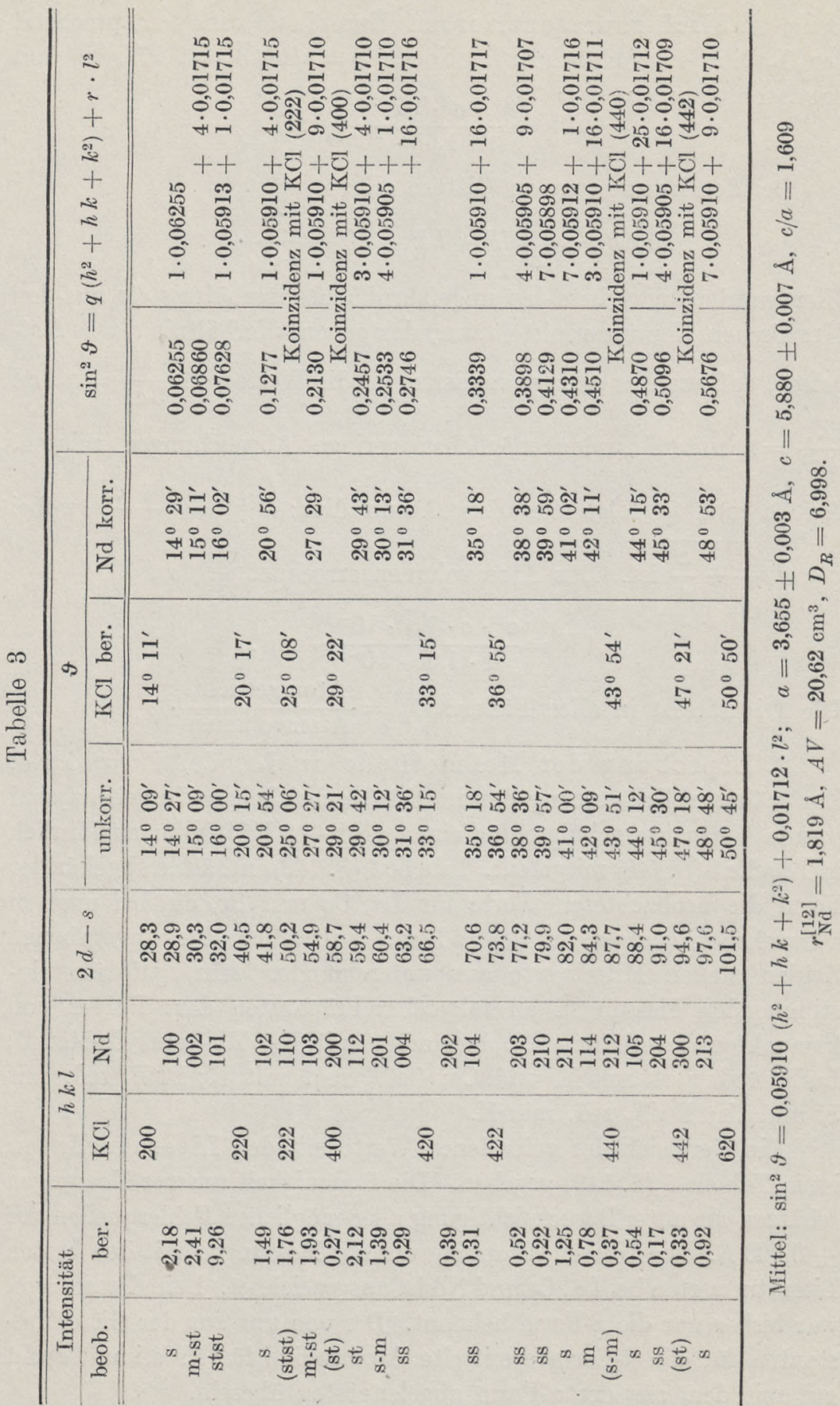


W. Klemm u. H. Bommer. Zur Kenntnis der Metalle der seltenen Erden 149

Tabelle 4

\begin{tabular}{|c|c|c|c|c|c|c|c|}
\hline \multirow{2}{*}{$\begin{array}{l}\text { Inten- } \\
\text { sität }\end{array}$} & \multirow{2}{*}{$h k l$} & \multicolumn{2}{|c|}{ Lanthan } & \multicolumn{2}{|c|}{ Cer } & \multicolumn{2}{|c|}{ Praseodym } \\
\hline & & $\vartheta_{\text {korr. }}$ & $a$ & $\vartheta_{\text {korr. }}$ & $a$ & $\vartheta_{\text {korr. }}$ & $a$ \\
\hline $\begin{array}{l}\text { st } \\
\text { sss }\end{array}$ & $\begin{array}{l}111 \\
101\end{array}$ & $14^{0} 35^{\prime}$ & 5,294 & $\begin{array}{r}15^{\circ} 02^{\prime} \\
\text { he }\end{array}$ & 5,138 & $\begin{array}{ll}15^{\circ} & 00^{\prime}\end{array}$ & 5,150 \\
\hline $\mathrm{m}$ & 200 & $16^{0} 54^{\prime}$ & 5,294 & $17^{\circ} 26^{\prime}$ & 5,137 & $\mid 17^{\circ} 23^{\prime}$ & 5,151 \\
\hline m-st & 220 & $24^{0} 16^{\prime}$ & 28 & Ko & zidenz & nit $\mathrm{KCl}(22$ & \\
\hline sss & 103 & & & & gonale & $\mathrm{Ce}$ und $\mathrm{Pr}$ & \\
\hline st & 311 & $28^{\circ} 50^{\prime}$ & 5,292 & $29^{0} 46^{\prime}$ & 5,141 & $29^{\circ} 42^{\prime}$ & 5,151 \\
\hline s & 222 & $30^{\circ} 15^{\prime}$ & 5,2 & $31^{0} 15^{\prime}$ & 5,138 & $31^{0} 09^{\prime}$ & 5,153 \\
\hline ss & 400 & $35^{\circ} 30^{\prime}$ & 5,29 & Ko & zidenz & nit $\mathrm{KCl}(42$ & \\
\hline $\mathrm{m}$ & 331 & $\begin{array}{lll}39^{\circ} & 20^{\prime}\end{array}$ & 5,291 & $40^{\circ} \quad 42^{\prime}$ & 5,143 & $40^{\circ} 37^{\prime}$ & 5,152 \\
\hline $\mathrm{m}$ & 420 & $40^{\circ} 33^{\prime}$ & 5,293 & $42^{\circ} 00^{\prime}$ & 5,143 & $41^{0} 55^{\prime}$ & 5,151 \\
\hline $\mathbf{s}$ & 422 & $45^{0} 24^{\prime}$ & 5,2 & $47^{\circ} 09^{\prime}$ & 5,142 & $47^{\circ} 00^{\prime}$ & 5,154 \\
\hline$s-m$ & $511+333$ & $49^{\circ} \quad 02^{\prime}$ & 5,295 & $51^{\circ} 01^{\prime}$ & 5,143 & Koin. mit & $\mathrm{KCl}(620)$ \\
\hline ss & & $55^{0} 18^{\prime}$ & 5,295 & & & & \\
\hline $\mathrm{s}-\mathrm{m}$ & 531 & $\begin{array}{lll}59^{0} & 18^{\prime}\end{array}$ & 5,294 & & & & \\
\hline $\mathrm{s}$ & $600+442$ & $60^{\circ} \quad 40^{\prime}$ & , & & & & \\
\hline \multicolumn{4}{|c|}{$\begin{array}{l}\text { Mittel: } a=5,294 \pm 0,002 \AA \\
\text { Atomradius } r^{[12]}=1,872 \AA \\
\text { Atomvolumen } A V=22,48 \mathrm{~cm}^{2} \\
\text { Dichte } D_{R}=6,18\end{array}$} & \multicolumn{2}{|c|}{$\begin{array}{l}5,140 \pm 0,002 \AA \\
1,817 \AA \\
20,58 \mathrm{~cm}^{3} \\
6,81_{0}\end{array}$} & \multicolumn{2}{|c|}{$\begin{array}{l}5,151 \pm 0,002 \AA \\
1,821 \AA \\
20,71 \mathrm{~cm}^{3} \\
6,80_{5}\end{array}$} \\
\hline
\end{tabular}

Tabelle 5

\begin{tabular}{|c|c|c|c|c|c|c|c|c|c|}
\hline \multirow[b]{2}{*}{ Metall } & \multirow{2}{*}{$\begin{array}{l}\text { Gitter- } \\
\text { Typus }\end{array}$} & \multicolumn{4}{|c|}{ Literaturwert } & \multicolumn{4}{|c|}{ Hier gefunden } \\
\hline & & $a$ & $c$ & $c / a$ & $\begin{array}{l}\text { Atom- } \\
\text { radius }\end{array}$ & $a$ & $c$ & $c / a$ & $\begin{array}{l}\text { Atom- } \\
\text { radius }\end{array}$ \\
\hline$\alpha$-La & hex. P. & $\begin{array}{l}\left.3,72^{1}\right) \\
\left.3,754^{2}\right)\end{array}$ & $\begin{array}{l}6,06 \\
6,063\end{array}$ & $\begin{array}{l}1,63 \\
1,613\end{array}$ & & & & & \\
\hline$\beta$-La & kub. flz. & $\left.5,296^{3}\right)$ & - & - & 1,872 & 5,294 & - & - & 1,872 \\
\hline$\alpha-\mathrm{Ce}$ & hex. P. & $\left.3,65^{4}\right)$ & 5,96 & 1,63 & 1,81 & & & & \\
\hline$\beta$-Ce & kub. flz. & $\begin{array}{l}\left.5,12^{4}\right) \\
\left.5,143^{2}\right)\end{array}$ & - & $\overline{-}$ & $\begin{array}{l}1,81 \\
1,819\end{array}$ & & & & \\
\hline$\alpha-\operatorname{Pr}$ & hex. P. & $\begin{array}{l}5,140^{3} \\
3,657^{5}\end{array}$ & 5,924 & $\overline{1,620}$ & $\begin{array}{l}1,817 \\
1,824\end{array}$ & 5,140 & - & - & 1,817 \\
\hline$\beta-\operatorname{Pr}$ & kub. flz. & - & - & - & - & 5,151 & - & - & 1,821 \\
\hline$\alpha-\mathrm{Nd}$ & hex. P. & $\left.3,657^{2}\right)$ & 5,880 & 1,608 & 1,819 & 3,655 & 5,880 & 1,609 & 1,819 \\
\hline$\alpha-\mathrm{Er}$ & hex. P. & $\left.3,74^{6}\right)$ & 6,09 & 1,63 & 1,86 & 3,532 & 5,589 & 1,582 & 1,748 \\
\hline
\end{tabular}

1) I. C. Mo Lennan u. R. W. Me Kay, Trans. Roy. Soc. Canada (3) Sect. III, 24 (1930), 33; Chem. Zbl.. 1931, II, 2418.

2) L. L. QuinL, Z. anorg. u. allg. Chem. 208 (1932), 273.

$\left.{ }^{3}\right)$ E. ZnNTu u. S. Neumayer, Z. Elektrochem. 39 (1933), 85.

$\left.{ }^{4}\right)$ A. W. Hull, Physical Review 18 (1921), 88.

5) A. Rossi, Atti Rendic. Reale Accad. Naz. Lincei (6) 15 (1932), 298.

$\left.{ }^{6}\right)$ I. C. Mc.Lennan u. R. I. Monkmann, Trans. Roy. Soc. Canada (3)

Sect. III, 23 (1929), 255. 
Tabelle 6

\begin{tabular}{|c|c|c|c|c|c|c|c|}
\hline \multirow{2}{*}{$\begin{array}{c}\text { Inten- } \\
\text { sität } \\
\text { beob. }\end{array}$} & \multirow{2}{*}{$h k l$} & \multicolumn{2}{|r|}{ Gd } & \multicolumn{2}{|r|}{$\mathrm{Tb}$} & \multicolumn{2}{|r|}{ Dy } \\
\hline & & $\vartheta_{\text {korr. }}$ & $\begin{array}{r}q\left(h^{2}+h k+k^{2}\right) \\
+r \cdot l^{2}\end{array}$ & $\vartheta_{\text {korr. }}$ & $\begin{array}{r}q\left(h^{2}+h k+k^{2}\right) \\
+r \cdot l^{2}\end{array}$ & $\vartheta_{\text {korr. }}$ & $\begin{array}{r}q\left(h^{2}+h k+k^{2}\right) \\
+r \cdot l^{2}\end{array}$ \\
\hline s & 100 & $14^{0} 24^{\prime}$ & $1 \cdot 0,06185$ & $14^{0} 24^{\prime}$ & $1 \cdot 0,06185$ & $14^{0} 44^{\prime}$ & $1 \cdot 0,06468 ?$ \\
\hline $\mathrm{m}_{\mathrm{stat}}$ & 002 & $15^{0} 36^{\prime}$ & $+4 \cdot 0,01808$ & $15^{0} 45^{\prime}$ & $+4 \cdot 0,01842$ & $15^{0} 49^{\prime}$ & $+4 \cdot 0,01856$ \\
\hline ststst & 101 & $16^{0} 14^{\prime}$ & $\begin{array}{l}1 \cdot 0,06020+ \\
1 \cdot 0,01795\end{array}$ & $16^{0} 25^{\prime}$ & $\begin{array}{c}1 \cdot 0,06143+ \\
1 \cdot 0,01845\end{array}$ & $16^{0} 27^{\prime}$ & $\begin{array}{r}1 \cdot 0,06165+ \\
1 \cdot 0,01855\end{array}$ \\
\hline$m-s t$ & 102 & $21^{0} 17^{\prime}$ & $\begin{array}{r}1 \cdot 0,06016+ \\
4 \cdot 0,01790\end{array}$ & $21^{0} 35^{\prime}$ & $\begin{array}{r}1 \cdot 0,06140+ \\
4 \cdot 0,01848\end{array}$ & $21^{0} 38^{\prime}$ & $\begin{array}{r}1 \cdot 0,06168+ \\
4 \cdot 0,01856\end{array}$ \\
\hline st & 110 & koin. & mit $\mathrm{KCl}(222)$ & $25^{0} 24^{\prime}$ & $3 \cdot 0,06133$ & $25^{0} 29^{\prime}$ & $3 \cdot 0,06170$ \\
\hline st & 103 & $28^{0} 06^{\prime}$ & $\begin{array}{c}1 \cdot 0,06020+ \\
9 \cdot 0,01795\end{array}$ & $28^{\circ} 27^{\prime}$ & $\begin{array}{l}1 \cdot 0,06140+ \\
9 \cdot 0,01840\end{array}$ & $28^{0} 35^{\prime}$ & $\begin{array}{c}1 \cdot 0,06170+ \\
9 \cdot 0,01858\end{array}$ \\
\hline ss & 200 & koin. & mit $\mathrm{KCl}(400)$ & & & $29^{0} 48^{\prime}$ & $4 \cdot 0,06174$ \\
\hline stst & 112 & $30^{0} 09^{\prime}$ & $\begin{array}{c}3 \cdot 0,06021+ \\
4 \cdot 0,01791\end{array}$ & $30^{0} 31^{\prime}$ & $\begin{array}{c}3 \cdot 0,06143+ \\
4 \cdot 0,01840\end{array}$ & $30^{\circ} 36^{\prime}$ & $\begin{array}{c}3 \cdot 0,06165+ \\
4 \cdot 0,01854\end{array}$ \\
\hline m-st & $\begin{array}{l}201 \\
004\end{array}$ & $30^{0} 33^{\prime}$ & $\begin{array}{r}4 \cdot 0,06011+ \\
1 \cdot 0,01790\end{array}$ & $30^{0} 55^{\prime}$ & $\begin{array}{c}4 \cdot 0,06139+ \\
1 \cdot 0,01845\end{array}$ & $31^{0} 00^{\prime}$ & $\begin{array}{r}4 \cdot 0,06165+ \\
1 \cdot 0,01854\end{array}$ \\
\hline s & 202 & $33^{0} 48^{\prime}$ & $\begin{array}{r}4 \cdot 0,06009+ \\
4 \cdot 0,01789\end{array}$ & $34^{0} 25^{\prime}$ & $\begin{array}{r}4 \cdot 0,06141+ \\
4 \cdot 0,01845\end{array}$ & $34^{0} 30^{\prime}$ & $\begin{array}{c}4 \cdot 0,06166+ \\
4 \cdot 0,01855\end{array}$ \\
\hline ss & 104 & & & koin & mit $\mathrm{KCl}(422)$ & koin. & mit $\mathrm{KCl}(422)$ \\
\hline $\mathrm{s}-\mathrm{m}$ & 203 & $39^{0} 21^{\prime}$ & $\begin{array}{r}4 \cdot 0,06020+ \\
9 \cdot 0,01791\end{array}$ & $39^{0} 55^{\prime}$ & $\begin{array}{r}4 \cdot 0,06142+ \\
9 \cdot 0,01844\end{array}$ & $40^{\circ} 04^{\prime}$ & $\begin{array}{l}4 \cdot 0,06174+ \\
9 \cdot 0,01858\end{array}$ \\
\hline $\begin{array}{c}\text { ss } \\
\text { m-st }\end{array}$ & $\begin{array}{l}210 \\
211\end{array}$ & $41^{0} 27^{\prime}$ & $\begin{array}{c}7 \cdot 0,06018+ \\
1 \cdot 0,01790\end{array}$ & $42^{\circ} 01^{\prime}$ & $\begin{array}{c}7 \cdot 0,06152+ \\
1 \cdot 0,01845\end{array}$ & $\begin{array}{l}41^{0} 04^{\prime} \\
42^{0} 10^{\prime}\end{array}$ & $\begin{array}{l}7 \cdot 0,06165 \\
7 \cdot 0,06169+ \\
1 \cdot 0,01855\end{array}$ \\
\hline $\mathrm{m}$ & 114 & $43^{0} 06^{\prime}$ & $\begin{array}{c}3 \cdot 0,06020+ \\
16 \cdot 0,01789\end{array}$ & $43^{0} 50^{\prime}$ & $\begin{array}{c}3 \cdot 0,06145+ \\
16 \cdot 0,01846\end{array}$ & $43^{0} 59^{\prime}$ & $\begin{array}{c}3 \cdot 0,06170+ \\
16 \cdot 0,01857\end{array}$ \\
\hline ss & 212 & $44^{0} 36^{\prime}$ & $\begin{array}{r}7 \cdot 0,06020+ \\
4 \cdot 0,01790\end{array}$ & $45^{0} 11^{\prime}$ & $\begin{array}{c}7 \cdot 0,06138+ \\
4 \cdot 0,01842\end{array}$ & $45^{0} 20^{\prime}$ & $\begin{array}{c}7 \cdot 0,06166+ \\
4 \cdot 0,01855\end{array}$ \\
\hline $\mathrm{s}$ & 105 & $45^{0} 28^{\prime}$ & $\begin{array}{r}1 \cdot 0,06020+ \\
25 \cdot 0,01792\end{array}$ & $46^{0} 21^{\prime}$ & $\begin{array}{r}1 \cdot 0,06140+ \\
25 \cdot 0,01848\end{array}$ & $46^{0} 30^{\prime}$ & $\begin{array}{c}1 \cdot 0,06170+ \\
25 \cdot 0,01858\end{array}$ \\
\hline ss & 204 & & & koin. $n$ & nit $\mathrm{KCl}(600)(442)$ & koin. m & ait KCI $(600)(442)$ \\
\hline $\begin{array}{l}\text { ss } \\
\text { s-m }\end{array}$ & $\begin{array}{l}300 \\
213\end{array}$ & $\begin{array}{l}\text { koin. m } \\
49^{\circ} 45^{\prime}\end{array}$ & $\begin{array}{l}\text { ait } \mathrm{KCl}(600)(442) \\
7 \cdot 0,06018+\end{array}$ & & & $\begin{array}{r}48^{0} 10^{\prime} \mid \\
\text { koin. }\end{array}$ & $\begin{array}{l}9 \cdot 0,06168 \\
\text { mit } \mathrm{KCl}(620)\end{array}$ \\
\hline s & 302 & $51^{0} 35^{\prime}$ & $\begin{array}{c}9 \cdot 0,01792 \\
9 \cdot 0,06023+ \\
4 \cdot 0,01795 \\
\end{array}$ & & & $52^{0} 30^{\prime}$ & $\begin{array}{c}9 \cdot 0,06161+ \\
4 \cdot 0,01854 \\
\end{array}$ \\
\hline \multicolumn{3}{|c|}{$\frac{\lambda^{2}}{3 a^{2}}=0,06018$} & & \multicolumn{2}{|c|}{0,06140} & \multicolumn{2}{|c|}{0,06168} \\
\hline \multicolumn{3}{|c|}{$\frac{\lambda^{2}}{4 c^{2}}=0,01792$} & & \multicolumn{2}{|c|}{0,01845} & \multicolumn{2}{|c|}{0,01856} \\
\hline \multicolumn{4}{|c|}{$a=3,622 \pm 0,003 \AA$} & \multicolumn{2}{|c|}{$3,585 \pm 0,003 \AA$} & \multicolumn{2}{|c|}{$3,578 \pm 0,002 \AA$} \\
\hline \multicolumn{4}{|c|}{$c=5,748 \pm 0,005 \AA$} & \multicolumn{2}{|c|}{$5,664 \pm 0,005 \AA$} & \multicolumn{2}{|c|}{$5,648 \pm 0,005 \AA$} \\
\hline \multicolumn{4}{|c|}{$c / a=1,587$} & \multicolumn{2}{|c|}{1,580} & \multicolumn{2}{|c|}{1,579} \\
\hline \multicolumn{4}{|c|}{$r^{[12]}=1,794 \AA$} & \multicolumn{2}{|c|}{$1,773 \AA$} & \multicolumn{2}{|c|}{$1,769 \AA$} \\
\hline \multicolumn{4}{|c|}{$A V=19,79 \mathrm{~cm}^{3}$} & \multicolumn{2}{|c|}{$19,11 \mathrm{~cm}^{3}$} & \multicolumn{2}{|c|}{$18,97 \mathrm{~cm}^{3}$} \\
\hline \multicolumn{4}{|c|}{$D_{R}=7,948$} & \multicolumn{2}{|c|}{8,332} & \multicolumn{2}{|c|}{8,562} \\
\hline
\end{tabular}


W. Klemm u. H. Bommer. Zur Kenntnis der Metalle der seltenen Erden 151

Tabelle 6

\begin{tabular}{|c|c|c|c|c|c|}
\hline \multicolumn{2}{|r|}{ Er } & \multicolumn{2}{|r|}{$\operatorname{Tm}$} & \multicolumn{2}{|r|}{$\mathrm{Cp}$} \\
\hline$\vartheta_{\text {korr. }}$ & $\begin{aligned} q\left(h^{2}+h k\right. & \left.+k^{2}\right) \\
& +r \cdot l^{2}\end{aligned}$ & $\vartheta_{\text {korr. }}$ & $\begin{aligned} q\left(h^{2}+h k\right. & \left.+k^{2}\right) \\
& +r \cdot l^{2}\end{aligned}$ & $\vartheta_{\text {korr. }}$ & $\begin{aligned} q\left(h^{2}+h k\right. & \left.+k^{2}\right) \\
& +r \cdot l^{2}\end{aligned}$ \\
\hline $32^{\prime}$ & $1 \cdot 0,06297$ & $35^{\prime}$ & $1 \cdot 0,06339$ & $14^{0} 39^{\prime}$ & $1 \cdot 0,06397$ \\
\hline $58^{\prime}$ & $+4 \cdot 0,01892$ & $16^{\urcorner} 02^{\prime}$ & $+4 \cdot 0,01908$ & $16^{7} 04^{\prime}$ & $+4 \cdot 0,01915$ \\
\hline $16^{0} 40^{\prime}$ & $\begin{array}{r}1 \cdot 0,06330+ \\
1 \cdot 0,01895\end{array}$ & $16^{0} 44^{\prime}$ & $\begin{array}{r}1 \cdot 0,06375+ \\
1 \cdot 0,01915\end{array}$ & $16^{0} 47^{\prime}$ & $\begin{array}{r}1 \cdot 0,06416+ \\
1 \cdot 0,01920\end{array}$ \\
\hline $21^{0} 54^{\prime}$ & $\begin{array}{r}1 \cdot 0,06332+ \\
4 \cdot 0,01895\end{array}$ & $22^{\circ} 00^{\prime}$ & $\begin{array}{r}1 \cdot 0,06368+ \\
4 \cdot 0,01916\end{array}$ & $22^{0} 02^{\prime}$ & $\begin{array}{r}1 \cdot 0,06410+ \\
4 \cdot 0,01916\end{array}$ \\
\hline $25^{0} 51^{\prime}$ & $3 \cdot 0,06337$ & $25^{0} 54^{\prime}$ & $3 \cdot 0,06360$ & $26^{\circ} 00^{\prime}$ & $3 \cdot 0,06404$ \\
\hline $55^{\prime}$ & $\begin{array}{l}1 \cdot 0,06335+ \\
9 \cdot 0,01893\end{array}$ & $29^{\circ} 03^{\prime}$ & $\begin{array}{l}1 \cdot 0,06365+ \\
9 \cdot 0,01913\end{array}$ & $29^{\circ} 06^{\prime}$ & $\begin{array}{r}1 \cdot 0,06407+ \\
9 \cdot 0,01916\end{array}$ \\
\hline $30^{9} 13^{\prime}$ & $4 \cdot 0,06332$ & $30^{9} 17^{\prime}$ & $4 \cdot 0,06359$ & $30^{7} 25^{\prime}$ & $4 \cdot 0,06409$ \\
\hline $31^{\circ} 01^{\prime}$ & $\begin{array}{r}3 \cdot 0,06328+ \\
4 \cdot 0,01892\end{array}$ & $31^{\circ} 08^{\prime}$ & $\begin{array}{l}3 \cdot 0,06360+ \\
4 \cdot 0,01913\end{array}$ & $31^{0} 15^{\prime}$ & $\begin{array}{r}3 \cdot 0,06418+ \\
4 \cdot 0,01915\end{array}$ \\
\hline $31^{0} 27^{\prime}$ & $\begin{array}{r}4 \cdot 0,06332+ \\
1 \cdot 0,01895\end{array}$ & $31^{0} 33^{\prime}$ & $\begin{array}{r}4 \cdot 0,06366+ \\
1 \cdot 0,01914\end{array}$ & $31^{0} 39^{\prime}$ & $\begin{array}{r}4 \cdot 0,06404+ \\
1 \cdot 0,01915\end{array}$ \\
\hline $35^{0} 01^{\prime}$ & $\begin{array}{l}4 \cdot 0,06336+ \\
4 \cdot 0,01895\end{array}$ & $35^{0} 07^{\prime}$ & $\begin{array}{l}4 \cdot 0,06363+ \\
4 \cdot 0,01912\end{array}$ & $35^{0} 13^{\prime}$ & $\begin{array}{l}4 \cdot 0,06400+ \\
4 \cdot 0,01913\end{array}$ \\
\hline $37^{0} 16^{\prime}$ & $1 \cdot 0,06330+16 \cdot 0,01896$ & $37^{0} 25^{\prime}$ & $1 \cdot 0,06360+16 \cdot 0,01910$ & $37^{0} 30^{\prime}$ & $1 \cdot 0,06404+16 \cdot 0,01913$ \\
\hline $40^{3} 37^{\prime}$ & $\begin{array}{r}4 \cdot 0,06331+ \\
9 \cdot 0,01895\end{array}$ & $40^{\circ} 47^{\prime}$ & $\begin{array}{r}4 \cdot 0,06364+ \\
9 \cdot 0,01911\end{array}$ & $40^{\top} 53^{\prime}$ & $\begin{array}{r}4 \cdot 0,06401+ \\
9 \cdot 0,01915\end{array}$ \\
\hline $41^{0} 41^{\prime}$ & $7 \cdot 0,06318$ & $41^{0} 51^{\prime}$ & $7 \cdot 0,06357$ & $42^{0} 03^{\prime}$ & $7 \cdot 0,06407$ \\
\hline $42^{0} 48^{\prime}$ & $\begin{array}{r}7 \cdot 0,06325+ \\
1 \cdot 0,01895\end{array}$ & $42^{\circ} 57^{\prime}$ & $\begin{array}{r}7 \cdot 0,06360+ \\
1 \cdot 0,01912\end{array}$ & $43^{\circ} 12^{\prime}$ & $\begin{array}{r}7 \cdot 0,06421+ \\
1 \cdot 0,01915\end{array}$ \\
\hline $44^{0} 39^{\prime}$ & $\begin{array}{r}3 \cdot 0,06335+ \\
16 \cdot 0,01899\end{array}$ & $44^{0} 51^{\prime}$ & $\begin{array}{r}3 \cdot 0,06365+ \\
16 \cdot 0,01914\end{array}$ & $44^{0} 57^{\prime}$ & $3 \cdot 0,06410+$ \\
\hline $46^{0} 03^{\prime}$ & $\begin{array}{r}7 \cdot 0,06322+ \\
4 \cdot 0,01895\end{array}$ & $46^{0} 12^{\prime}$ & $\begin{array}{r}7 \cdot 0,06352+ \\
4 \cdot 0,01909\end{array}$ & $46^{0} 24^{\prime}$ & $\begin{array}{r}7 \cdot 0,06399+ \\
4 \cdot 0,01915\end{array}$ \\
\hline Kom. & mit $\mathrm{KCl}(600)(442)$ & sUin & mit $\mathrm{KCl}(600)(442)$ & koin. & mit $\mathrm{KCl}(600)(442)$ \\
\hline $48^{\circ} 18^{\prime}$ & $4 \cdot 0,06330+16 \cdot 0,01901$ & $48^{0} 30^{\prime}$ & $4 \cdot 0,06368+16 \cdot 0,01915$ & & \\
\hline $48^{0} 59^{\prime}$ & $9 \cdot 0,06326$ & $49^{\prime} 08^{\prime}$ & $9 \cdot 0,06355$ & $49^{\circ} 22^{\prime}$ & $9 \cdot 0,06400$ \\
\hline $51^{0} 33^{\prime}$ & $\begin{array}{r}7 \cdot 0,06321+ \\
9 \cdot 0,01895\end{array}$ & $51^{0} 45^{\prime}$ & $\begin{array}{r}7 \cdot 0,06354+ \\
9 \cdot 0,01909\end{array}$ & $51^{0} 56^{\prime}$ & $\begin{array}{r}7 \cdot 0,06391+ \\
9 \cdot 0,01915\end{array}$ \\
\hline $53^{0} 25^{\prime}$ & $\begin{array}{r}9 \cdot 0,06322+ \\
4 \cdot 0,01895 \\
\end{array}$ & $53^{0} 37^{\prime}$ & $\begin{array}{r}9 \cdot 0,06353+ \\
4 \cdot 0,01909 \\
\end{array}$ & $53^{0} 55^{\prime}$ & $\begin{array}{r}9 \cdot 0,06412+ \\
4 \cdot 0,01915 \\
\end{array}$ \\
\hline
\end{tabular}

0,06330

0,06362

0,06410

0,01895

0,01912

$3,532 \pm 0,002 \AA$

$5,589 \pm 0,005 \AA$

1,582

$1,748 \AA$

$18,29 \mathrm{~cm}^{3}$

$3,523 \pm 0,002 \AA$

$5,564 \pm 0,005 \AA$

1,580

$1,742 \AA$

$18,12 \mathrm{~cm}^{3}$

9,346

0,01915

$3,509 \pm 0,003 \AA$

$5,559 \pm 0,005 \AA$

1,584

1,737 $\AA$

$17,96 \mathrm{~cm}^{3}$

9,740 
3. Die Strukturen von Eu, Gd, Tb, Dy, Er, Tm, Yb und Cp. Auf die soeben beschriebene Methode ließen sich von allen uns zur Verfügung stehenden $\operatorname{Erden}^{\mathbf{1}}$ ), deren Strukturen bisher nicht bekannt waren, die Gitter der Metalle ermitteln.

a) Von den untersuchten Erden kristallisieren hexagonal in angenähert dichter Packung: Gd, Tb, Dy, Er, Tm und Cp. Die Daten für diese Elemente sind in Tabelle 6 (S. 150-151) zusammengestellt.

Für diese Elemente sind noch folgende Bemerkungen erforderlich:

1. Die angegebenen Genauigkeiten sind die der Messungen. Nun standen uns, wie S. 141 beschrieben, beim Thulium und Cassiopeium und besonders beim Terbium nur angenähert reine Präparate zur Verfügung. Infolgedessen dürften die für diese Elemente ermittelten Gitterkonstanten nur angenähert richtig sein. Der Fehler kann aber nicht groß sein. Wir kommen auf diese Frage S. 157 noch einmal zurück.

2) Bei einem Terbiumpräparat beobachteten wir neben den Linien der hexagonalen Form auch schwach die stärksten des kubisch-flächenzentrierten Gitters. Vgl. S. 160.

3. Für Erbium liegt bereits eine Strukturbestimmung von J.C. MAC LENNAN und R. J. MonkmaN ${ }^{2}$ ) vor. Die Ergebnisse dieser Bestimmung sind mit unseren unvereinbar. Wie sich in $\mathrm{C}$ zeigen wird, paßt der $r$-Wert dieser Autoren in keiner Weise in den allgemeinen Verlauf der Atomradien. An der Richtigkeit des Befundes von MAC LENNAN und MoNKMANN hat auch schon W. BILTZ ${ }^{3}$ ) Zweifel geäußert.

b) Kubisch-flächenzentriert kristallisiert das Ytterbium. Die Ergebnisse sind in Tabelle 7 zusammengestellt.

Tabelle 7

$\mathrm{Yb}$ (Co $K \alpha$-Strahlung)

\begin{tabular}{|c|c|c|c|c|c|c|c|}
\hline $\begin{array}{c}\text { Intensität } \\
\text { beob. }\end{array}$ & $\begin{array}{l}\text { Indizes } \\
h k l\end{array}$ & $\vartheta_{\text {korr. }}$ & $\begin{array}{c}\text { Gitter- } \\
\text { konstante } \\
a\end{array}$ & $\begin{array}{c}\text { Intensität } \\
\text { beob. }\end{array}$ & $\begin{array}{l}\text { Indizes } \\
h k l\end{array}$ & $\vartheta_{\text {korr. }}$ & $\begin{array}{c}\text { Gitter- } \\
\text { konstante } \\
a\end{array}$ \\
\hline st st & 111 & $\left\{\begin{array}{c}\text { Koin- } \\
\text { zidenz }\end{array}\right\}$ & $\left.\mathrm{KCl}\left[200^{*}\right)\right]$ & $\begin{array}{c}\text { ss } \\
\text { m-st }\end{array}$ & $\begin{array}{l}400 \\
331\end{array}$ & $\begin{array}{l}40^{\circ} 48^{\prime} \\
45^{0} 25^{\prime}\end{array}$ & $\begin{array}{l}5,469 \\
5,469\end{array}$ \\
\hline m-st & 200 & $19^{\circ} 05^{\prime}$ & 5,465 & m-st & 420 & $46^{0} 55^{\prime}$ & 5,471 \\
\hline st & 220 & $27^{0} 33^{\prime}$ & 5,464 & m-st & 422 & $53^{0} 08^{\prime}$ & 5,471 \\
\hline st st & 311 & $32^{0} 49^{\prime}$ & 5,468 & st & $511 ; 333$ & $58^{\circ} 03^{\prime}$ & 5,471 \\
\hline $\mathrm{m}$ & 222 & $34^{0} 29^{\prime}$ & 5,465 & s & 440 & $67^{\circ} 23^{\prime}$ & 5,475 \\
\hline \multicolumn{8}{|c|}{ Mittel: $a=5,468 \pm 0,002 \AA$} \\
\hline
\end{tabular}

c) Kubisch-raumzentriertes Gitter fanden wir beim Europium. Vgl. Tabelle 8 (S. 153).

1) Bis auf Sm; vgl. S. 157.

2) J. C. Mac Lennan u. R. J. Monkman, Trans. Roy. Soc. Canada (3), - Sect. III, 23 (1929), 255.

3) W. Brutz, Raumchemie der festen Stoffe. Leipzig 1934, S. 18. 
W. Klemm u. H. Bommer. Zur Kenntnis der Metalle der seltenen Erden 153

\section{Tabelle 8}

Eu (Co $K \alpha$-Strahlung)

\begin{tabular}{c|c|c|c|c|c|c|c}
\hline $\begin{array}{c}\text { Intensität } \\
\text { beob. }\end{array}$ & $\begin{array}{c}\text { Indizes } \\
h k l\end{array}$ & $\vartheta_{\text {korr. }}$ & $\begin{array}{c}\text { Gitter- } \\
\text { konstante } \\
a\end{array}$ & $\begin{array}{c}\text { Intensität } \\
\text { beob. }\end{array}$ & $\begin{array}{c}\text { Indizes } \\
h k l\end{array}$ & $\vartheta_{\text {korr. }}$ & $\begin{array}{c}\text { Gitter- } \\
\text { konstante } \\
a\end{array}$ \\
\hline \hline ststst & 110 & $16^{0} 02^{\prime}$ & 4,575 & st & 321 & $47^{0} 01^{\prime}$ & 4,571 \\
s-m & 200 & $22^{0} 59^{\prime}$ & 4,576 & sss & 400 & & \\
st st & 211 & $28^{0} 35^{\prime}$ & 4,575 & m & $114 ; 330$ & $56^{0} 03^{\prime}$ & 4,571 \\
s & 220 & $33^{\circ} 33^{\prime}$ & 4,573 & s & 402 & $60^{0} 59^{\prime}$ & 4,570 \\
m & 301 & $38^{0} 10^{\prime}$ & 4,572 & s-m & 332 & $66^{0} 33^{\prime}$ & 4,568 \\
ss & 222 & $42^{0} 35^{\prime}$ & 4,574 & & & & \\
\hline
\end{tabular}

Mittel: $a=4,573 \pm 0,002 \AA$

$$
r^{[8]}=1,980 \AA . \quad r^{[12]}=2,042 \AA . \quad A V=29,00 . \quad D_{R}=5,24_{4}
$$

4. Zusammenstellung der Ergebnisse. Tabelle 9 gibt eine Übersicht über alle Gitter der Erdmetalle, die bisher röntgenographisch ermittelt sind. Wir haben in dieser Tabelle vorzugsweise unsere eigenen Messungen berücksichtigt. Nur die Werte für Yttrium und die $\alpha$-Formen des Lanthans, Cers und Praseodyms sind der Literatur entnommen. Die Auswertung dieser Tabelle wird im nächsten Abschnitt erfolgen.

\section{Tabelle 9}

\begin{tabular}{|c|c|c|c|c|c|c|c|}
\hline Element & $\begin{array}{l}\text { Gitter- } \\
\text { typus }\end{array}$ & $a$ & $c$ & $c / a$ & $\begin{array}{c}\text { Atom- } \\
\text { radius } \\
\text { f. Ko.-Z.12 }\end{array}$ & $\begin{array}{l}\text { Atom- } \\
\text { volumen } \\
\text { in } \mathrm{cm}^{3}\end{array}$ & Dichte \\
\hline$\left.\alpha-\mathrm{La}^{1}\right)$ & hex. P. & 3,754 & 6,063 & 1,613 & 1,870 & 22,43 & $6,19_{4}$ \\
\hline$\beta$-La & kub. flz. & 5,294 & & & 1,872 & 22,48 & $6,18_{0}$ \\
\hline$\left.\alpha-\mathrm{Ce}^{2}\right)$ & hex. P. & 3,65 & 5,96 & 1,63 & 1,81 & 20,7 & 6,78 \\
\hline$\beta$ - $\mathrm{Ce}$ & kub. flz. & 5,140 & - & - & 1,817 & 20,58 & $6,81_{0}$ \\
\hline$\left.\alpha-\operatorname{Pr}^{3}\right)$ & hex. P. & 3,657 & 5,924 & 1,62 & 1,824 & 20,79 & $6,77_{7}$ \\
\hline$\beta-\mathrm{Pr}$ & kub. flz. & 5,151 & - & - & 1,821 & 20,71 & $6,80_{5}$ \\
\hline $\mathrm{Nd}$ & hex. P. & 3,655 & 5,880 & 1,609 & 1,819 & 20,62 & $6,99_{8}$ \\
\hline $\begin{array}{c}61 \\
\text { Sm }\end{array}$ & $\bar{?}$ & - & - & - & - & $\Xi$ & - \\
\hline $\mathrm{Eu}$ & kub. rz. & 4,573 & - & - & 2,042 & 29,00 & $5,24_{1}$ \\
\hline $\mathrm{Gd}$ & hex. P. & 3,622 & 5,748 & 1,587 & 1,794 & 19,79 & $7,94_{3}$ \\
\hline $\mathrm{Tb}$ & hex. P. & 3,585 & 5,664 & 1,580 & 1,773 & 19,11 & $8,33_{2}$ \\
\hline Dy & hex. P. & 3,578 & 5,648 & 1,579 & 1,769 & 18,97 & $8,56_{2}$ \\
\hline $\begin{array}{l}\mathrm{Ho} \\
\mathrm{Er}\end{array}$ & hex. P. & $3 \overline{532}$ & $5, \overline{589}$ & 1,582 & 1,748 & 18,29 & $9, \overline{16}$ \\
\hline $\mathrm{Tm}$ & hex. P. & 3,523 & 5,564 & 1,580 & 1,742 & 18,12 & $9,34_{6}$ \\
\hline $\mathrm{Yb}$ & kub. flz. & 5,468 & - & - & 1,933 & 24,76 & $7,01_{0}^{\circ}$ \\
\hline Cp & hex. P. & 3,509 & 5,559 & 1,584 & 1,737 & 17,96 & $9,74_{0}$ \\
\hline$\left.Y^{4}\right)$ & hex. P. & 3,663 & 5,814 & 1,588 & 1,814 & 20,46 & 4,34 \\
\hline
\end{tabular}

1) Vgl. S. 149, L. L. QUILL.

2) Vgl. S. 149, A. W. HuLL.

3) Vgl. S. 149, A. Rossi.

4) L. L. OunL, Z. anorg. u. allg. Chem. 208 (1932), 59. 
Hier möchten wir nur auf den Gang der $c / a-W e r t e$ der hexagonalen Gitter hinweisen. Diese sind bei den Anfangsgliedern der Cer-Erden am größten, sie fallen dann beim Neodym und Gadolinium deutlich ab und nehmen schließlich vom Terbium bis zum Cassiopeium einen praktisch konstanten Wert an.

\section{Auswertung der Ergebnisse}

1. Die Atomvolumenkurve. Zeichnet man die Atomvolumenkurve für die seltenen Erdmetalle ${ }^{1}$ ) (vgl. Fig. 5), so erkennt man deutlich einen periodischen Verlauf. Während man ins-

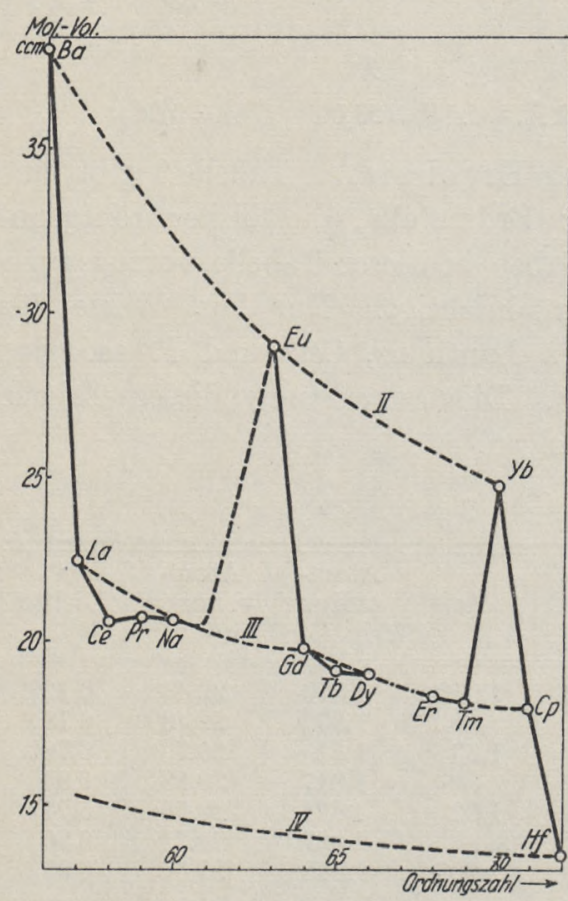

Fig. 5. Atomvolumenkurve gesamt einen ziemlich gleichmäßigen Abfall vom Lanthan zum Cassiopeium feststellen kann (Lanthanidenkontraktion), fallen Europium und Ytterbium durch sehr hohe, Cer, Praseodym und Terbium durch etwas zu niedrige Werte auf. Mit diesem periodischen Verlauf erinnert die Atomvolumenkurve der seltenen Erden sehr an die Atomvolumenkurve aller chemischen Elemente, die bekanntlich Lothar Meyer zur Aufstellung des periodischen Systems der Elemente führte.

Bei den seltenen Erden liegt nun ein periodisches System für die Ione $n^{2}$ ) bereits vor, das von W. KLемм insbesondere auf Grund des Auftretens

höherer und niedrigerer Wertigkeiten aufgestellt worden ist. Fig. 6, die einer Abhandlung von G. JАNтsch und W. Kцемм ${ }^{3}$ ) entnommen ist, möge die Verhältnisse noch einmal in Erinnerung rufen. Atomtheoretisch ausgezeichnet sind die Konfigurationen des $\mathrm{La}^{3+}$ (Edelgaskonfiguration), $\mathrm{Gd}^{3+}$ (halbabgeschlossene Untergruppe)

1) Auf die Darstellung der Atomradienkurve ist verzichtet, da sie der Atomvolumenkurve vollkommen entspricht.

$\left.{ }^{2}\right)$ W. Kuemm, Z. anorg. u. allg. Chem. 184 (1929), 345; 187 (1930), 29; 209 (1932), 321; G. JANTSCH u. W. KLемM, Z. anorg. u. allg. Chem. 216 (1933), 80.

${ }^{3}$ ) G. JANTsch u. W. KLemm, Z. anorg. u. allg. Chem. 216 (1933), 80. 
und $\mathrm{Cp}^{3+}$ (ganz abgeschlossene Untergruppe). Die benachbarten Elemente suchen nun diese abgeschlossenen Konfigurationen zu erreichen, die vorhergehenden durch Bildung von Verbindungen mit 2-wertigen Ionen $(\mathrm{Eu}$ und $\mathrm{Yb})$, die folgenden durch Verbindungen mit 4-wertigen Ionen (Ce und $\mathrm{Tb}$ ). Bei den zwei Stellen von den ausgezeichneten Zuständen entfernt stehenden Elementen zeigt sich in zwei Fällen noch eine T'endenz zur Annäherung an diese ausgezeichneten Konfigurationen (2-Wertigkeit des $\mathrm{Sm}$ und 4-Wertigkeit des Pr).

Es liegt nahe, ähnliche Gründe auch für die Besonderheiten der Atomvolumenkurve der seltenen Erden verantwortlich zu machen. Legt man als das einfachste Schema die Vorstellung zugrunde, daß die Metalle der seltenen Erden aus positiven Ionen und einem Elek-

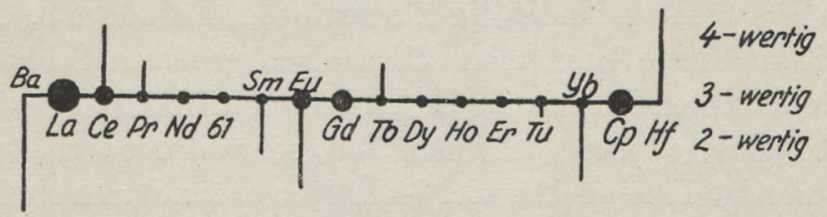

Fig. 6. Schematische Darstellung des Auftretens 2- und 4-wertiger Verbindungen in der Reihe der seltenen Erden, wobei die Striche nach oben das Auftreten 4-wertiger, die Striche nach unten das Auftreten 2-wertiger Verbindungen bedeuten. Die Länge der Striche gibt ein ungefähres Maß der Beständigkeit, die Größe der Punkte deutet die relative Stabilität der Elektronenkonfiguration an, die beim $\mathrm{Me}^{3+}$ vorliegt

tronengas aufgebaut sind, so würden die nur 3-wertig auftretenden seltenen Erden 3 fach positive geladene Ionen und je 3 Elektronen pro Atom im Elektronengas erwarten lassen. Dies würde vermutlich zu einem gleichmäßigen Abfall der Atomvolumenkurve führen. Bilden dagegen einzelne Elemente im metallischen Zustand 2-wertige Ionen, so sollte dies in Analogie zum Barium höhere Átomvolumina ergeben, während sich umgekehrt das Vorhandensein 4-wertiger Ionen durch niedrigere Werte für das Atomvolumen kennzeichnen sollte. Legt man diese Annahme zugrunde, so wäre beim Europiumund Ytterbiummetall auf die Anwesenheit 2-wertiger Ionen im Gitter $\mathrm{zu}$ schließen, beim Cer und angedeutet auch beim Praseodym und Terbium auf die Anwesenheit 4-wertiger İonen. Dies entspräche aber genau den anomalen Wertigkeiten, die man bei salzartigen Verbindungen gefunden hat. Daraus folgt: Die auf Grund der Wertigkeitsverhältnisse begründete Systematik der Ionen der seltenen Erden gilt auch für den metallischen Zustand. 
Sie prägt sich im Gang der Atomvolumenkurve sogar besonders deutlich aus.

2. Einzelheiten. Zu diesem allgemeinen Ergebnis mögen noch einige Einzelheiten hinzugefügt werden.

a) In der Fig. 5 sind drei gestrichelte Kurven eingetragen, die mit II, III und IV bezeichnet sind. Diese Kurven bedeuten folgendes: Die Kurve III verbindet diejenigen Elemente, für die mit großer Wahrscheinlichkeit 3-wertige Ionen im Metall anzunehmen sind. Wie man erkennt, verläuft die Kurve nicht ganz gleichmäßig; sie besteht vielmehr aus zwei Hälften: La-Gd einerseits, Gd-Cp andererseits. Die Kurve III spiegelt somit auch in ihrem feineren Verlauf die immer wieder hervortretende Zweiteilung der seltenen Erden wieder.

Die Kurve II verbindet die Elemente $\mathrm{Ba}, \mathrm{Eu}$ und $\mathrm{Yb}$. Aus dem Gang dieser Kurve kann man schon einen gewissen Schluß darauf ziehen, ob bei den Elementen $\mathrm{Eu}$ und $\mathrm{Yb}$ im Metall nur oder überwiegend 2-wertige Ionen vorhanden sind, oder ob nur verhältnismäßig wenig 2-wertige Ionen neben viel 3-wertigen anzunehmen sind. Durch den Gesamtverlauf der Kurve II ist der Schluß nahegelegt, daß ganz überwiegend 2-wertige Ionen vorhanden sind.

Anders ist es bei den Elementen, bei denen die Bildung 4-wertiger Ionen im Metallgitter anzunehmen ist, also bei Ce, Pr und Tb. Enthielten diese Elemente im metallischen Zustand nur 4-wertige Ionen, so sollte man Atomvolumina erwarten, die etwa auf der durch das Atomvolumen des Hafniums bestimmten Kurve IV lägen. Aus der tatsächlichen Lage folgt, daß Ce nur einen geringen, $\mathrm{Pr}$ und $\mathrm{Tb}$ nur einen verschwindend kleinen Bruchteil an 4-wertigen Ionen enthalten können.

Sind die eben genannten Abschätzungen der Anteile der 2- und 4-wertigen Ionen richtig, so würde daraus das allgemeine Ergebnis folgen, daß im Metall die Bildung 2-wertiger Ionen mehr begünstigt ist als die 4-wertiger Ionen. Dieses Ergebnis steht nicht vereinzelt da. Z. B. haben E. VoGт und andere ${ }^{1}$ ) sehr verdünnte feste Lösungen von Chrom, Mangan, Eisen, Kobalt in unmagnetischen Metallen wie Kupfer, Silber und Gold magnetisch untersucht. Dabei haben sich magnetische Momente für die gelösten Metalle ergeben, die in großen Zügen der Annahme entsprechen, daß 2-wertige positive Ionen gebildet werden. Auch hier ist also offenbar die Bildung 2-wertiger Ionen im metallischen System begünstigt.

1) Vgl.W.KLемм, Magnetochemie.Leipzig 1936, S. 207 ff., insbesondere S. 212. 
Bei den reinen Metallen der eben genannten Übergangselemente ist ein entsprechender Schluß aus den magnetischen Messungen nicht abzuleiten, da hier infolge der Wechselwirkungen zwischen den Außenelektronen [Ferromagnetismus und Antiferromagnetismus $\left.\left.{ }^{1}\right)\right]$ die Verhältnisse allzu kompliziert sind. Jedoch gibt es auch hier ein Anzeichen dafür, daß der 2-fach ionisierte Zustand beim Aufbau der Metalle eine besondere Rolle spielt. Das Mangan besitzt als Metall eine deutliche Sonderstellung. Das Gitter ist anders als das der Nachbarn Chrom und Eisen, das Atomvolumen ist etwas größer, das Produkt aus Ausdehnungskoeffizient und Schmelzpunkt ${ }^{2}$ ) besitzt beim Mangan (und übrigens auch beim Rhenium) ein Maximum. Nun nimmt das Mangan nur in der Reihe der 2-wertigen Ionen eine Sonderstellung: ein (halbbesetzte Elektronenkonfiguration), nicht aber z. B. in der Reihe der 3-wertigen Ionen, bei denen diese Halbbesetzung der $3 d$-Schale beim $\mathrm{Fe}^{3+}$ erreicht wird. Die genannten Sonderheiten des metallischen Mangans sind also nur zu verstehen, wenn den Me ${ }^{2+}$-Ionen eine besondere Bedeutung für den Aufbau dieser Metalle zukommt.

b) Bezüglich der Elemente, von denen wir nur angenähert reine Präparate zur Verfügung hatten und derjenigen, deren Strukturen noch nicht festgestellt werden konnten, ist folgendes zu sagen:

«) Die für Terbium in Frage kommenden Verunreinigungen (in erster Linie Gd und Dy) könnten nur eine Erhöhung des Atomvolumens bewirkt haben. Der genaue Wert für Terbium dürfte etwas niedriger liegen. Der Einfluß der Verunreinigung kann aber nicht groß sein.

$\beta$ ) Ein geringer $\mathrm{Cp}$-Gehalt des verwendeten Thuliums würde ohne Einfluß sein, eine kleine Menge $\mathrm{Yb}$ könnte möglicherweise zu einer Gitteraufweitung führen. Der für Thulium gefundene Atomradius ist also ein Maximalwert.

$\gamma)$ Da das von uns verwendete Cassiopeium nur etwas Thulium enthielt, aber frei von Ytterbium war, ist die Verunreinigung für die Größe des Atomvolumens ohne Bedeutung.

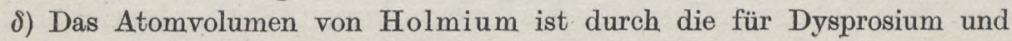
Erbium gemessenen Werte mit großer Sicherheit festgelegt.

ع) Für das Atomvolumen des Samariums läßt sich eine Voraussage aus der S. 156 dargestellten allgemeinen Beziehung, daß die Tendenz zur Bildung 2-wertiger Ionen im metallischen Zustande besonders groß ist, ableiten. Die Untersuchung der salzartigen Verbindungen hat ergeben, daß das Samarium ebenfalls 2-wertige Verbindungen bildet, die aber wesentlich unbeständiger sind als die des $\mathrm{Yb}$ und besonders die des Eu. Es ist danach anzunehmen, daß das Atomvolumen des Samariummetalls etwas größer sein wird, als der Kurve III entspricht, aber deutlich kleiner als das des Europiums. Dieser Annahme scheint allerdings die Dichtemessung von Muthmann und Werss ${ }^{3}$ ), die zu einem Atomvolumen von nur $19-20 \mathrm{~cm}^{3}$ führt, zu widersprechen. Diese Messung ist aber vermutlich an einem so unreinen Präparat gemacht, daß ihr entscheidender Wert nicht beizumessen ist. Für unsere Annahme spricht aber das magnetische Verhalten, wie unter D. näher gezeigt werden wird.

1) Vgl. W. KLwMm, Magnetochemie. Leipzig 1936, S. 207.

$\left.{ }^{2}\right)$ W. KLEмm, Z. anorg. u. allg. Chem. 198 (1931), 178.

${ }^{3}$ ) W. Muthmann u. L. Weiss, Lieb. Ann. 331 (1904), 1. 
Ђ) Für das Element 61 lassen sich sichere Voraussagen zur Zeit nicht machen. Sein Atomvolumen entspricht wahrscheinlich der Kurve III, vielleicht liegt es eine Kleinigkeit höher.

c) Bei der Untersuchung der anomalen Wertigkeiten hatte sich ergeben, daß in der ersten Hälfte der seltenen Erden die Tendenz zur Bildung 4- und 2-wertiger Verbindungen wesentlich größer ist als in der zweiten ${ }^{1}$ ) (vgl. dazu Fig. 6, S. 155). Das gleiche kann man auch bei den Metallen feststellen. So liegt das Atomvolumen des Terbiums nur ganz schwach unterhalb der Kurve III, das des Cers dagegen recht deutlich. Während ferner das Praseodym noch merklich von dieser Kurve abweicht, liegt das ihm in der zweiten Hälfte entsprechende Dysprosium genau auf der Kurve. Ferner bildet Thulium im metallischen Gitter keinerlei 2-wertige Ionen, während beim Samarium das Vorhandensein einer erheblichen Menge 2-wertiger Ionen durch die magnetischen Messungen erwiesen ist (vgl. S. 169).

d) Die Lanthanidenkontraktion ist bei den Metallen nicht sehr groß. Die Differenz der Atomvolumina von La und Cp beträgt $4,5 \mathrm{~cm}^{3}$. W. Bistz gibt für die Differenz der Ionenvolumina der beiden Elemente $3 \mathrm{~cm}^{3}$ an, also einen nur wenig kleineren Wert ${ }^{2}$ ). Diese Abstufung ist ähnlich wie bei den entsprechenden Elementen der Übergangsreihe, die ebenfalls 3 -wertige Ionen besitzen. Z. B. beträgt die Differenz der Atomvolumina von $\mathrm{Y}$ und In $5 \mathrm{~cm}^{3}$, die der Ionenvolumina ungefähr $2 \mathrm{~cm}^{3}$.

Bei den Metallen niederer Wertigkeiten sind die Unterschiede viel größer; so beträgt die Differenz zwischen $\mathrm{Sr}$ und $\mathrm{Cd} 20,5 \mathrm{~cm}^{3}$ für das Atom-, aber nur $5 \mathrm{~cm}^{3}$ für das Ionenvolumen. Dem entspricht bei den seltenen Erdmetallen, daß die Differenz zwischen Ba- und Yb-Metall ungefähr $13 \mathrm{~cm}^{3}$ beträgt, die zwischen $\mathrm{BaCl}_{2}$ und $\mathrm{YbCl}_{2}$ aber nur $5 \mathrm{~cm}^{3}$.

e) Vergleicht man das Atomvolumen des Yttriummetalls mit den Werten für die anderen Erdmetalle, so findet man, daß es dem des Neodyms sehr nahe steht. Dagegen liegen die Atomvolumina von Dysprosium und Erbium, deren 3-wertige Ionen etwa die gleiche Größe haben wie $\mathrm{Y}^{3+}$, wesentlich tiefer. Die Größe des Elektronengases [Differenz von Atomvolumen und Ionenvolumen ${ }^{3}$ )] bei $0^{0}$ absolut beträgt, wenn man die Biltz'schen Ioneninkremente zugrunde legt, für Yttrium 14,2 $\mathrm{cm}^{3}$, für Erbium dagegen nur $12,3 \mathrm{~cm}^{3}$.

1) G. JANTSCH u. W. Krem, Z. anorg. u. allg. Chem. 216 (1933), 80.

$\left.{ }^{2}\right)$ Bei den Atomradien beträgt der Unterschied zwischen La und Cp nur $0,135 \AA$, während die Differenz der Radien von $\mathrm{La}^{3+}$ und $\mathrm{Cp}^{3+} 0,23 \AA$ beträgt. Hier kommt das langsame Absinken bei den Metallen ganz besonders deutlich zum Ausdruck.

3) Vgl. W. BiLtz, Raumchemie der festen Stoffe. Leipzig 1934, S. 206. 
Dieser Unterschied ist leicht zu verstehen. Das Yttriumion enthält wesentlich weniger Elektronen als etwa das Erbiumion. Wenn trotzdem das Ionenvolumen dieser beiden Ionen annähernd gleich ist, so bedeutet dies, daß die kontrahierende Wirkung des Erbiumatomkerns auf die Elektronenhülle des Erbiumions wesentlich größer ist als die des Yttriumkerns auf seine Außenelektronen. Infolgedessen nimmt es nicht Wunder, daß auch die komprimierende Wirkung des Erbiumkerns auf das Elektronengas im Erbiummetall größer ist als die des Yttriumkerns auf das Elektronengas im Yttriummetall.

f) Schließlich sind noch einige Bemerkungen über die Gitterstrukturen erforderlich. Vorherrschend ist die hexagonal-dichte Packung; daneben findet man die $\beta$-Formen mit kubisch-dichter Packung beim La, $\mathrm{C} \theta$ und $\mathrm{Pr}$ und vielleicht auch beim Tb. Die hexagonal-dichte Struktur ist nicht beobachtet beim Eu und $\mathrm{Yb}$, von denen das zweite kubisch-flächenzentriert, das erste kubischraumzentriert kristallisiert.

Daß es sich bei diesem Auftreten verschiedener Gittertypen um die Auswirkung ganz bestimmter Gesetzmäßigkeiten handelt, erkennt man aus folgendem: In der nachstehenden Tabelle 10 sind die Gitter einiger Elemente mit edelgasähnlichen Ionen im Metallverband nach dem Atomvolumen geordnet zusammengestellt. Es ergibt sich aus dieser Tabelle, daß das Auftreten der verschiedenen Strukturen bei gegebener Valenzelektronenzahl offenbar in erster Linie durch die Größe des Atomvolumens bestimmt ist: Die weiträumigsten Elemente kristallisieren kubisch-raumzentriert, die nächst engeren kubisch-flächenzentriert, die engsten schließlich hexagonal-dicht ${ }^{1}$ ).

Tabelle 10

\begin{tabular}{|c|c|c|c|c|c|c|c|c|}
\hline & $\begin{array}{l}\text { Atom- } \\
\text { Vol. }\end{array}$ & $\begin{array}{c}\text { Gitter- } \\
\text { typ }\end{array}$ & & $\begin{array}{l}\text { Atom- } \\
\text { Vol. }\end{array}$ & Gittertyp & & $\begin{array}{l}\text { Atom- } \\
\text { Vol. }\end{array}$ & Gittertyp \\
\hline \multirow{3}{*}{$\begin{array}{l}\mathrm{Cu} \\
\mathrm{Au} \\
\mathrm{Ag}\end{array}$} & 7,1 & kub. flz. & \multirow{4}{*}{$\begin{array}{l}\mathrm{Be} \\
\mathrm{Zn} \\
\mathrm{Cd} \\
\mathrm{Mg}\end{array}$} & \multirow{4}{*}{$\begin{array}{r}4,9 \\
9,1 \\
13,0 \\
14,0\end{array}$} & hex. d. & B & 3,3 & hex. \\
\hline & 10,2 & & & & " , & $\mathrm{Al}$ & 10,0 & kub. flz. \\
\hline & 10,3 & , & & & $"$, & $\left.\mathrm{Sc}^{2}\right)$ & 14,5 & hex. d. \\
\hline \multirow{5}{*}{$\begin{array}{l}\mathrm{Li} \\
\mathrm{Na} \\
\mathrm{K} \\
\mathrm{Rb} \\
\mathrm{Cs}\end{array}$} & \multirow{5}{*}{$\begin{array}{l}13,0 \\
23,7 \\
45,4 \\
55,8 \\
71,0\end{array}$} & \multirow{5}{*}{$\begin{array}{cc}\text { kub. rz. } \\
, \quad, \quad \\
, \quad, \\
", \quad " \\
", \quad\end{array}$} & & & ", & $\mathrm{Y}$ & 20,5 & , \\
\hline & & & Hg & 14,1 & hex. eig. Typ. & \multirow{4}{*}{ La } & \multirow{4}{*}{22,5} & \multirow{4}{*}{$\begin{array}{l}\text { hex. d. } \\
\text { u. kub. flz. }\end{array}$} \\
\hline & & & $\mathrm{Ca}$ & 26,0 . & $\begin{array}{l}\text { hex. d. } \\
\text { u. kub. flz. }\end{array}$ & & & \\
\hline & & & $\mathrm{Sr}$ & 33,7 & kub. flz. & & & \\
\hline & & & $\mathrm{Ba}$ & 38,0 & kub. rz. & & & \\
\hline
\end{tabular}

1) Eine Ausnahme bildet in der 3. Gruppe das Aluminium, das kubisch flächenzentriert kristallisiert, obwohl es ein kleineres Volumen besitzt als das hexagonale Scandium. Das Verhalten des Aluminiums würde ohne weiteres verständlich, wenn mạn annähme, daß es im Gitter nicht 3-, sondern 1-wertige Ionen bildet, was manche Besonderheiten des Aluminiums, z. B. seinen niedrigen Schmelzpunkt, erklären würde.

$\left.{ }^{2}\right)$ Privatmitteilung von W. Fischer, vgl. dieses Heft S. 54. 
Bei den Metallen der seltenen Erden betrachten wir die Elemente mit verschiedener Ladung der positiven Ionen im Metall gesondert.

a) 2-wertige Ionen: Der Gitterwechsel zwischen $\mathrm{Eu}$ und $\mathrm{Yb}$ entspricht den allgemeinen Erfahrungen: Das weiträumige Element kristallisiert kubisch-raumzentriert, das engere kubisch-flächenzentriert.

Die Grenze zwischen diesen beiden Gittertypen liegt nicht ganz an derselben Stelle wie bei den Erdalkalimetallen, bei denen $\operatorname{Sr}\left(\mathrm{AV}=33,7 \mathrm{~cm}^{3}\right)$ bereits kubischflächenzentriert ist, während $\mathrm{Eu}\left(\mathrm{AV}=29,0 \mathrm{~cm}^{3}\right)$ noch kubisch raumzentriert ist.

ß) 3-wertige Ionen: Hier kommt das größte Element (La) sowohl kubisch-flächenzentriert als auch hexagonal-dicht vor, während die übrigen Elemente Nd, Gd, Dy, (Ho), Er, Tm und Cp nur in hexagonaler Packung kristallisieren.

$\gamma) 3$ - und 4-wertige Ionen nebeneinander: Hier scheint die kubisch-flächenzentrierte Form etwas mehr bevorzugt zu sein; denn $\mathrm{Ce}$ und $\mathrm{Pr}$ kommen, obwohl sie im Atomvolumen kleiner sind als $\mathrm{Nd}$, nicht nur hexagonal-dicht, sondern auch kubisch-flächenzentriert vor. Ferner sind auch beim $\mathrm{Tb}$ Andeutungen für die Existenz einer kubischflächenzentrierten Form gefunden worden (vgl. S. 152).

Dieser Einfluß der Me $\mathrm{M}^{4}$-Ionen war zu erwarten; denn auch das Thorium kristallisiert kubisch-flächenzentrisch, obwohl sein Atomvolumen etwas kleiner ist als das des Neodyms.

\section{Magnetische Untersuchungen}

Es war erwünscht, die aus der Atomvolumenkurve abgeleiteten Schlüsse über den Ionisierungszustand der seltenen Erden im Metall durch magnetische Messungen zu stützen. Dies kann in allen Fällen, in denen die Annahme zulässig ist, daß die Metalle aus positiven Ionen und einem Elektronengas aufgebaut sind, durch magnetische Untersuchungen geschehen. Es wird dann nämlich die magnetische Suszeptibilität praktisch nur durch das Moment der positiven Ionen bestimmt, während der Diamagnetismus der positiven Ionen und der geringe, temperaturunabhängige Paramagnetismus des Elektronengases ganz zurücktreten. Es war allerdings nicht anzunehmen, daß dieses einfache Schema in allen Fällen ausreichen würde. Vielmehr mußte damit gerechnet werden, daß durch stärkere Wechselwirkungen zwischen den Atomen Komplikationen auftraten, insbesondere ferromagnetische Erscheinungen. Nach derartigen Wechselwirkungen zu suchen, war eine weitere Aufgabe dieser Untersuchung.

Während der Durchführung unserer Versuche erschien eine Mitteilung von Urbain, Trombe und $\mathrm{WeIss}^{1}$ ), in der gezeigt wurde,

1) G. Urbain, F. Trombe u. P. Weiss, Compt. rend. 200 (1935), 2132. 
daß das metallische Gadolinium ferromagnetisch ist, eine Angabe, die wir voll bestätigen können. Damit erschien die Untersuchung der anderen Yttererden ganz besonders dringlich.

Die bisher in der Literatur verzeichneten Angaben über das magnetische Verhalten der Erdmetalle betreffen, wenn man von der Untersuchung eines gänzlich undefinierten Erbiums durch OwEN ${ }^{1}$ ) absieht, nur die Elemente La, Ce, Pr und Nd. Diese Untersuchungen litten alle darunter, daß es bei der Herstellung von kompaktem Material durch Schmelzelektrolyse offenbar sehr schwierig ist, Eisen, d. h. ferromagnetische Verunreinigungen, ganz auszuschließen. Infolgedessen stimmen die erhaltenen Werte, auf die wir im einzelnen nicht eingehen wollen ${ }^{2}$ ), zwar bei Zimmertemperatur leidlich überein, weichen aber bei tiefen Temperaturen stark voneinander ab.

\section{Die Messungsergebnisse}

Wir selbst haben nicht die kompakten Metalle, sondern die bei der Reduktion der Chloride mit Alkalimetall erhaltenen pulverförmigen Gemische aus Erdmetall +3 Alkalichlorid untersucht. Dies brachte den Vorteil mit sich, daß Verunreinigungen, besonders solche von ferromagnetischen Stoffen, weitgehend vermieden werden konnten. Dementsprechend erwiesen sich die magnetischen Suszeptibilitäten unserer Präparate bei höheren Temperaturen in allen Fällen als unabhängig von der Feldstärke. Bei tiefen Temperaturen fanden auch wir bei einer Reihe der untersuchten Präparate Feldștärkenabhängigkeit. Wir werden später Gründe für die Annahme anführen, daß diese Feldstärkenabhängigkeit den Erden selbst eigentümlich ist und nur unwesentlich durch Verunreinigungen beeinflußt sein dürfte.

Bei der Wiedergabe unserer Meßergebnisse haben wir darauf verzichtet, alle an den verschiedenen Präparaten gemessenen Einzelwerte aufzuführen. Wir geben vielmehr für jedes Element nur Mittelwerte. Diese sind aber jeweils an mindestens zwei unabhängig dargestellten Präparaten gewonnen, deren Einzelwerte auf wenigstens $3 \%$ übereinstimmten. Ferner begnügen wir uns, die Atomsuszeptibilitäten anzugeben, da die für das Gemisch aus Alkalichlorid und Erdmetall direkt gemessenen Gramm-Suszeptibilitäten wenig charakteristisch sind. Diese Ergebnisse sind in Tabelle 11 zusammengestellt.

1) M. Owen, Ann. Phys. 37 (1912), 657.

2) M. Owen, Ann. Phys. 37 (1912), 657; F. Trombe, Compt. rend. 198 (1934), I591; V. J. Drozzina u. R. J. JaANus, Phys. Ztschr. Sowjetunion 9 (1936), 72 ; L. F. Wereschtschagtn, L. W. Schubnikow u. B. J. Laserew, Phys. Ztschr. Sowjetunion, Juni (1936), 107.

z. anorg. u. allg. Chem. Bd. 231. 
162 Zeitschrift für anorganische und allgemeine Chemie. Band 231. 1937

Zu dieser Tabelle möchten wir noch auf zwei Einzelheiten hinweisen.

a) Es kam uns bei unseren Messungen weniger darauf an, die einzelnen Elemente nach den verschiedensten Richtungen hin in besonders genauer Weise zu untersuchen, als vielmehr darauf, eine allgemeine Übersicht zu

Tabelle 11

Gemessene Suszeptibilitäten $\chi_{\text {At. }}$.

\begin{tabular}{|c|c|c|c|c|c|c|c|}
\hline Element & $T^{0}$ & $\chi_{\text {Atom }} \cdot 10^{6}$ & Feldstärke & Element & $T^{0}$ & $\chi_{\text {Atom }} \cdot 10^{6}$ & Feldstärke \\
\hline La & $\begin{array}{r}90 \\
291 \\
\end{array}$ & $\begin{array}{l}+125 \\
+125\end{array}$ & $\begin{array}{c}\text { unabh. } \\
\text {, }\end{array}$ & \multirow[t]{6}{*}{$\mathrm{Tb}$} & \multirow{6}{*}{$\begin{array}{l}273 \\
292 \\
341 \\
406 \\
493 \\
555\end{array}$} & $\begin{array}{r}327000 \\
\sim 324000\end{array}$ & $\begin{array}{c}2050 \\
\infty\end{array}$ \\
\hline \multirow[t]{3}{*}{$\left(e^{1}\right)$} & 90 & 4480 & unabh. & & & 146000 & unabh. \\
\hline & 195 & 3160 & " & & & 115000 & $"$ \\
\hline & 291 & 2340 & $"$ & & & 74700 & , \\
\hline \multirow[t]{3}{*}{ Pr } & 90 & 14350 & unabh. & & & $\begin{array}{l}49400 \\
35100\end{array}$ & $"$ \\
\hline & 195 & 6600 & $"$ & & & $\begin{array}{l}35100 \\
29100\end{array}$ & ," \\
\hline & 293 & 4380 & $"$ & \multirow{22}{*}{ Dy } & \multirow{5}{*}{114} & 1760000 & 350 \\
\hline \multirow[t]{3}{*}{$\mathrm{Nd}$} & 90 & 16700 & unabh. & & & 1680000 & 540 \\
\hline & 195 & 7920 & ", & & & $\sim 1500000$ & $\infty$ \\
\hline & 293 & 5350 & ", & & & 1460000 & 350 \\
\hline \multirow[t]{4}{*}{$\mathrm{Sm}$} & 90 & 4940 & 2050 & & & 1430000 & 540 \\
\hline & & 4550 & 3640 & & \multirow{3}{*}{136} & $\sim 1350000$ & $\infty$ \\
\hline & 195 & $\begin{array}{r}4000 \\
2180\end{array}$ & $\infty$ & & & 1000000 & 350 \\
\hline & 291 & $\begin{array}{l}2180 \\
1820\end{array}$ & $\begin{array}{c}\text { unabh. } \\
\text {, }\end{array}$ & & & $\begin{array}{r}942000 \\
\sim 850000\end{array}$ & 540 \\
\hline \multirow[t]{7}{*}{$\mathrm{Eu}^{2}$ ) } & 90 & 385000 & 540 & & \multirow[t]{4}{*}{159} & 688000 & 350 \\
\hline & & 307000 & 1060 & & & 668000 & 540 \\
\hline & & 245000 & 2050 & & & 630000 & 1060 \\
\hline & & $\sim 150000$ & $\infty$ & & & $\sim 560000$ & \\
\hline & 167 & 56300 & unabh. & & \multirow{2}{*}{195} & 355000 & 540 \\
\hline & 195 & 46000 & $"$ & & & 348000 & 1060 \\
\hline & 293 & 30400 & " & & & $\sim 340000$ & $\infty$ \\
\hline \multirow[t]{4}{*}{ Gd } & 418 & 64500 & unabh. & & \multirow[t]{2}{*}{273} & 112000 & 1060 \\
\hline & 485 & 40500 & , & & & 108000 & 2050 \\
\hline & 495 & 38400 & " & & \multirow{5}{*}{$\begin{array}{l}291 \\
337 \\
396 \\
454 \\
508\end{array}$} & $\sim 104000$ & \\
\hline & 623 & 23300 & $"$ & & & 102000 & unabh. \\
\hline \multirow[t]{12}{*}{$\mathrm{Tb}$} & \multirow[t]{3}{*}{90} & \multirow{12}{*}{\begin{tabular}{|r|}
3070000 \\
2850000 \\
$\sim 2400000$ \\
2590000 \\
2350000 \\
2165000 \\
$\sim 1800000$ \\
1240000 \\
1180000 \\
$\sim 1050000$ \\
536000 \\
508000 \\
$\sim 460000$ \\
335000 \\
329000
\end{tabular}} & 350 & & & 97200 & , \\
\hline & & & 540 & & & 59200 & , \\
\hline & & & $\infty$ & & & $\begin{array}{l}48000 \\
41100\end{array}$ & ״, \\
\hline & \multirow[t]{3}{*}{195} & & $\begin{array}{l}350 \\
540\end{array}$ & \multirow[t]{5}{*}{ Er } & \multirow{4}{*}{90} & 458000 & " \\
\hline & & & 800 & & & 399000 & 800 \\
\hline & & & $\infty$ & & & 355000 & 1060 \\
\hline & 216 & & 540 & & & $\sim 200000$ & $\infty$ \\
\hline & & & $\begin{array}{c}1060 \\
\infty\end{array}$ & & $\begin{array}{l}195 \\
291\end{array}$ & $\begin{array}{l}72000 \\
44500\end{array}$ & unabh. \\
\hline & \multirow[t]{2}{*}{228} & & 540 & \multirow[t]{3}{*}{$\left.\operatorname{Tm}^{3}\right)$} & 90 & 89500 & unabh. \\
\hline & & & 1060 & & 195 & 39000 & , \\
\hline & \multirow[b]{2}{*}{237} & & $\infty$ & & 291 & 25600 & , \\
\hline & & & $\begin{array}{r}540 \\
1060\end{array}$ & $\mathrm{Yb}$ & $\begin{array}{r}90 \\
292\end{array}$ & 640 & unabh. \\
\hline & & & & & & & ," \\
\hline ) 1 & Vgl. & Fig. 10. & & & & & \\
\hline 3) & & & & & & & \\
\hline
\end{tabular}


erhalten. Infolgedessen sind unsere Messungen nach manchen Seiten hin unvollständig. Z. B. ergab sich, daß einzelne Elemente bei tiefen Temperaturen einen außerordentlich hohen Magnetismus zeigten, so daß wir sie mit unserer Meßeinrichtung bei manchen Temperaturen nur bei verhältnismäßig kleinen Feldstärken (unter 1000 Gauss) messen konnten.

b) Ferner sind wir einigen Sonderheiten im magnetischen Verhalten noch nicht nachgegangen. So hat z. B. F. Trombe bei der magnetischen Untersuchung des metallischen Cers eine Art Hysteresis gefunden, die bei steigender und fallender Temperatur gemessenen $1 / \chi$ - $T$-Kurven decken sich nicht. Wir haben, wie Fig. 7 zeigt, bei der Untersuchung unserer Cerpräparate das gleiche gefunden. Die Dauer

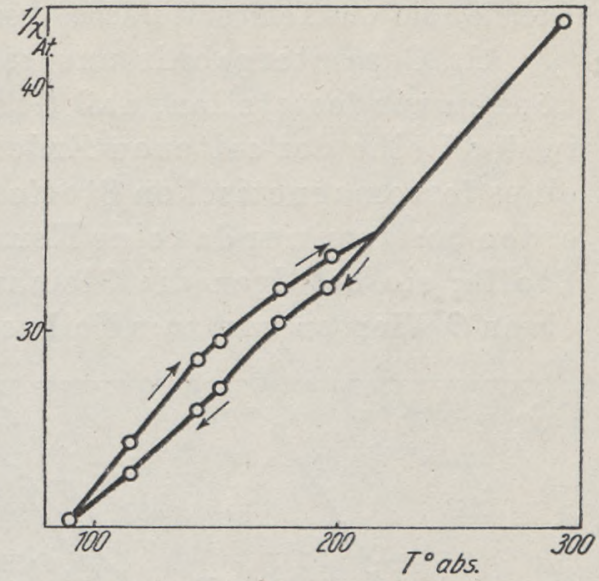

Fig. 7. Temperatur-Hysterese des Ce-Metalls des Erwärmens von $90-195^{\circ}$ abs. betrug etwa 4 Stunden. Die nähere Aufklärung dieser Verhältnisse haben wir für später zurückgestellt.

2. Das Auftreten ferromagnetischer Elemente in der Reihe der seltenen Erden

Bei der Besprechung der Ergebnisse behandeln wir zunächst die Frage nach dem Auftreten ferromagnetischer Elemente in der Reihe der seltenen Erden. Die Auswirkungen des Ferromagnetismus sind bekanntlich verschieden, je nachdem in welchem Temperaturgebiet man sich befindet. Unterhalb der sogenannten CurIETemperatur findet man sehr hohe Suszeptibilitätswerte, die außerdem feldstärkenabhängig sind. Reicht das zur Messung benutzte äußere Magnetfeld zur Sättigung aus, so sind die $\chi$-Werte der äußeren Feldstärke annähernd umgekehrt proportional. Oberhalb der Curie-Temperatur ist die Suszeptibilität unabhängig von der Feldstärke. Die Temperaturabhängigkeit ist dann durch die Gleichung $\chi(T-\Theta)=C$ bestimmt. Die durch diese Gleichung gegebene „,paramagnetische CurIE-Temperatur" $\Theta$ fällt bei den meisten ferromagnetischen Stoffen mit der direkt beobachteten ,ferromagnetischen CurIE-Temperatur" praktisch zusammen ${ }^{1}$ ). Die Gültigkeit der eben

1) Für einen idealen ferromagnetischen Stoff verlangt die Theorie ferner, daß die Momente, die man aus der (ferromagnetischen) Magnetisierungsintensität bei unendlicher Feldstärke einerseits aus der Temperaturabhängigkeit der Suszeptibilitäten im paramagnetischen Gebiet andererseits berechnet, in einem ganz bestimmten 
genannten Gleichung prüft man am einfachsten dadurch, daß man die $1 / \chi$-Werte gegen die Temperatur aufträgt. Dann ergibt sich eine Gerade, die die Temperaturachse bei der Temperatur $\Theta$ schneidet.

Als Gesamtergebnis unserer magnetischen Messungen können wir feststellen, daß Gadolinium das einzige Metall in der Reihe der seltenen Erden ist, das alle Kennzeichen eines ferromagnetischen Stoffes zeigt. Alle anderen Yttererden besitzen nur das eine Kennzeichen ferromagnetischer Stoffe: sie befolgen die Gleichung $\chi(T-\Theta)=C$ mit positiven $\Theta$-Werten von zum Teil recht erheblicher Größe. Da-

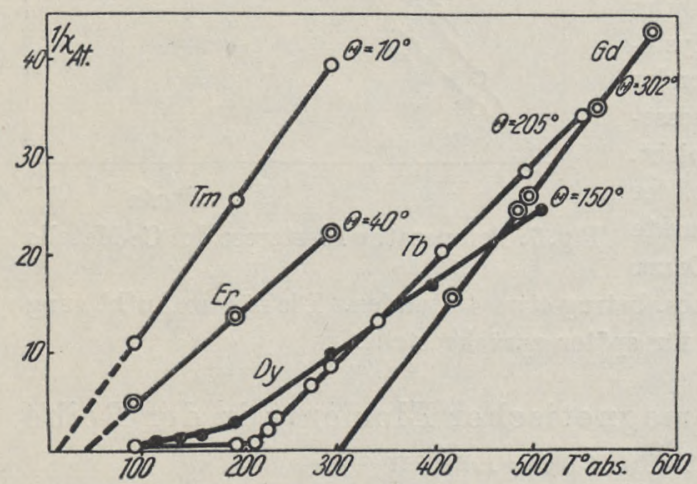

Fig. 8. $1 / \chi_{\text {At. }}-T$-Kurven

für die Metalle der Yttererden

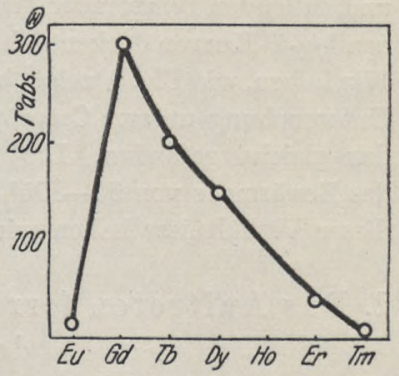

Fig. 9. „CURIE-Temperaturen“ für die Metalle der Yttererden

gegen zeigen sie unterhalb dieser $\Theta$-Temperatur keine vollständige Ausbildung von Ferromagnetismus, sondern nur Übergangszustände zwischen paramagnetischem und ferromagnetischem Verhalten.

Betrachten wir zunächst die Verhältnisse oberhalb der $\Theta$-Temperatur. Fig. 8 zeigt die Verhältnisse für die Elemente Gd, Tb, Dy, Er und Tm. Man erkennt überall den geradlinigen Verlauf der $1 / \chi-T$ Kurven bis zumeist sehr kleinen $1 / \chi$-Werten hin. Die aus den Schnittpunkten dieser Kurven mit der Temperaturachse ermittelten $\Theta$-Werte

Verhältnis zueinander stehen. Ist nämlich das Gesamtspinmoment $S$, so sollte gelten: $\mu_{\text {para }}=2 \cdot \sqrt{S(S+1)}$ und $\mu_{\text {ferro }}=2 \cdot S . \quad($ Vgl. dazu W. KLEMm, Magnetochemie, Leipzig 1936, S. 107.) Diese Forderung der Theorie war bisher noch nie erfüllt. Das erste Beispiel, für das sie wirklich zutrifft, ist das metallische Gadolinium. Für dieses fanden Urbarn, Trombe und WeIss $\mu_{\text {ferro }}=7,15, \mu_{\text {para }}=7,95$ Magnetonen. Da das $\mathrm{Gd}^{3+}$-Ion ein Gesamtspinmoment $S$ von $7 / 2 \cdot h / 2 \pi$ besitzt, so berechnen sich $\mu_{\text {ferro }}=7,0$ und $\mu_{\text {para }}=7,94$ Magnetonen, also mit dem experimentellen Befunde sehr gut übereinstimmende Werte. 
sind in Fig. 9 zusammengestellt. Darin ist auch das Europium mit aufgenommen worden, auf das wir weiter unten zurückkommen werden. Es ergibt sich für $\operatorname{die} \Theta$-Werte ein Maximum beim $\mathrm{Gd}^{1}$ ) und nach der Seite des Europiums ein schroffer, nach den Elementen höherer Ordnungszahl zu ein flacherer Abfall.

Daß unterhalb der CuRIE-Temperatur der vollständige ferromagnetische Zustand nicht erreicht wird, erkennt man bereits aus Fig. 8. Diese zeigt, da $\beta$ sich der Verlauf der $1 / \chi-T$-Kurve bei tiefen Temperaturen zwar sprunghaft ändert, daß sich aber dabei die Suszeptibilitäten noch in mäßigen Grenzen halten. Lägen wirklich ferromagnetische Stoffe vor, so würden in unserem Maßstabe die $1 / \chi$-Werte mit der Abszisse zusammenfallen. Ferner müßten die den ferromagnetischen Substanzen im Sättigungsgebiet zukommenden $\chi$-Werte den Meßfeldstärken angenähert umgekehrt proportional sein. Wie Tabelle 11 zeigt, ist dies auch nicht angenähert der Fall. Es wird notwendig sein, das Verhalten dieser Elemente bei hohen Feldstärken zu untersuchen, damit man entscheiden kann, ob diese hier gefundenen Erscheinungen darauf zurückzuführen sind, daß es sich um Ferromagnetika mit einer sehr flachen $J$-H-Kurve ${ }^{2}$ ) handelt oder ob die Suszeptibilitäten bei sehr hohen Feldstärken einem Grenzwert zustreben. Im letzteren Falle würden ganz neuartige Erscheinungen vorliegen.

Man könnte einwenden, daß die bei den Nachbarelementen des Gadoliniums beobachteten Erscheinungen alle darauf zurückzuführen seien, daß die Präparate Gd-haltig sind. Diese Annahme ist zwar nicht mit völliger Sicherheit zu entkräften. Wir werden sie dadurch zu entscheiden versuchen, $d a ß$ wir möglichst hochgereinigte Erden verschiedener Herkunft untersuchen und prüfen, ob wir stets dieselben $\Theta$-Werte finden. Schon jetzt läßt sich aber sagen, daß viel gegen die Annahme einer Verunreinigung durch Gd spricht. Einmal bilden benachbarte seltene Erden Mischkristalle miteinander, und man weiß aus dem Verhalten des Eisens und anderer ferromagnetischer Elemente, daß Mischkristalle mit einem nichtferromagnetischen Metall als Grundsubstanz nicht ferromagnetisch sind. Ferner waren die von uns untersuchten Präparate von sehr verschiedener Reinheit. Das Terbium z. B. dürfte mindestens $5 \%$ Gadolinium enthalten, während unser Dysprosium bestimmt sehr viel weniger enthielt. Trotzdem ist der Abfall der Werte vom Gadolinium zum Dysprosium ganz gleichmäßig.

Ein besonders eigenartiges magnetisches Verhalten liegt beim Europium vor. Dieses zeigt nach der $1 / \chi-T$-Kurve im paramagnetischen Gebiete nur einen verhältnismäßig niedrigen $\Theta$-Wert $\left(+15^{0}\right.$ absolut), dagegen finden sich schon

1) Auch bei den ferromagnetischen Gliedern der Übergangselemente findet man ein ähnliches Verhalten. Hier besitzt das Co eine wesentlich höhere CURIETemperatur $\left(1130^{\circ} \mathrm{C}\right)$ als das $\mathrm{Fe}\left(680^{\circ}\right)$ und das $\mathrm{Ni}\left(370^{\circ}\right)$.

2) $J=$ Magnetisierungsintensität, $H=$ Feldstärke. 
unterhalb $150^{\circ}$ absolut deutliche Abweichungen von geradlinigen Verlauf der $1 / \chi$-T-Kurve und gleichzeitig damit Feldstärkenabhängigkeit (vgl. Fig. 10). Es erscheint uns auch hier kaum wahrscheinlich, daß dieser Abfall auf irgendeine Verunreinigung zurückzuführen ist. Jedoch sind noch genauere Untersuchungen erforderlich.

Das bei den Metallen der Yttererden beobachtete magnetische Verhalten scheint uns von besonderem Interesse für die Theorie des Ferromagnetismus. Für das Zustandekommen von Ferro-

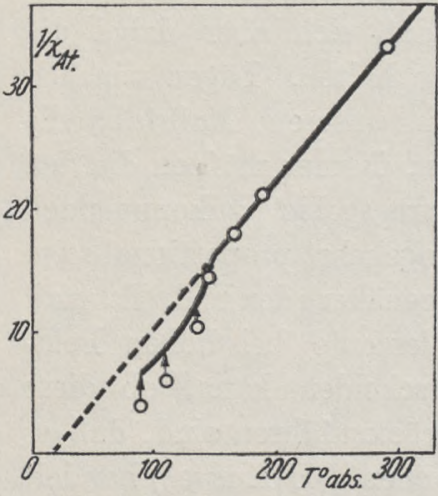

Fig. 10. $1 / \chi_{\text {At }}-T$-Kurve für Eu-Metall magnetismus sah man bisher zwei Bedingungen als besonders wesentlich an $\left.{ }^{1}\right)$ :

a) Es muß eine unabgeschlossene Schale mit hoher Nebenquantenzahl vorliegen.

b) Der Gitterabstand muß groß sein gegenüber dem Radius dieser Schale.

Beide Bedingungen sind bei allen Lanthaniden mit Ausnahme von La, Yb und $\mathrm{Cp}$ erfüllt; die $4 \mathrm{f}$-Elektronenschale ist unabgeschlossen, der Radius dieser Schale ist klein gegenüber den Atomabständen. Trotzdem ist nur ein einziges Element wirklich ferromagnetisch, nämlich das in der Mitte der Reihe stehende Gadolinium. Es ist also offenbar notwendig, daß die Zahl der für Atombindungen mit parallelem Spin zur Verfügung stehenden Elektronen der unabgeschlossenen Schale einen ganz bestimmten Wert hat, der irgendwie mit der Zahl der Gitternachbarn in gesetzmäßiger Beziehung steht. Merkwürdig ist dabei, daß zwischen den 7 ferromagnetisch wirksamen Elektronen des Gadolinium-Ions und der Zahl der Nachbaratome, die ja 12 beträgt, kein einfacher zahlenmäßiger Zusammenhang besteht.

Sehr interessant ist fernerhin das Europium. Die in ihm vorhandenen 2-wertigen Ionen besitzen ja die gleiche Elektronenkonfiguration wie die im metallischen Gd enthaltenen 3-wertigen Gd-Ionen. Trotzdem zeigt Eu im Gegensatz zum ferromagnetischen Gd höchstens ganz schwache Andeutungen von Ferromagnetismus. Der Unterschied liegt offenbar in zwei Dingen begründet:

a) Die Zahl der Nachbarn ist hier nicht 12, sondern 8; das Verhältnis der Zahl der 4 f-Elektronen zu der der Gitternachbarn ist also anders.

1) Vgl. W. KLemм, Magnetochemie. Leipzig 1936, S. 134. 
b) Die Atomabstände sind wesentlich größer. Infolgedessen dürfte die zum Ferromagnetismus führende Austauschenergie sehr viel schwächer sein.

Es wäre sehr interessant, wenn es gelänge, Eu-Metall mit kubisch-flächenzentriertem Gitter darzustellen, da dann die Zahl der Nachbaratome ebensogroß würde wie beim hexagonalen Gadolinium; unterscheiden würden sich dann nü die Abstände. Ferner wollen wir Eu-haltige Mischkristalle untersuchen, sowohl solche mit Gd, als auch solche mit $\mathrm{Sm}$ und $\mathrm{Nd}$.

\section{Die Ionenmomente der seltenen Erdmetalle}

Zur Ermittelung der Ionenmomente und damit der Ionenladungen in den Metallen, die in Zusammenhang mit der Atomvolumenkurve besonders wichtig sind, betrachten wir die Elemente Lanthan bis Samarium, bei denen ferromagnetische Erscheinungen überhaupt nicht auftreten, getrennt von denen der Yttererden.

a) Lanthan bis Samarium. Für Lanthan-Metall mit $\mathrm{La}^{3+}$ Ionen sollte man unmagnetisches Verhalten erwarten. In der Tat findet man auch nur einen sehr geringen Paramagnetismus.

Für das Cer hatten wir aus der Atomvolumenkurve geschlossen, daß sowohl 3-als auch 4-wertige Ionen vorliegen. Diese Annahme ist magnetisch leicht $\mathrm{zu}$ prüfen, da das $\mathrm{Ce}^{4+-}$-Ion kein magnetisches Moment besitzt, während dem $\mathrm{Ce}^{3+}$-Ion ein Moment von 2,56 Magnetonen zukommt. Berechnet man nach der Formel $\mu_{\text {eff. }}=2,84 \sqrt{\chi_{\text {At. }} \cdot T}$ die effektiven Momente für Ce-Metall, so erhält man folgende Werte:

$$
\begin{aligned}
& 90^{\circ} \text { abs. }: \mu_{\text {eff. }}=1,80 \\
& 195^{\circ} \text { abs. }: \mu_{\text {eff. }}=2,23 \\
& 291^{\circ} \text { abs. }: \mu_{\text {eff. }}=2,34,
\end{aligned}
$$

d.h. in der Tat Momente, die zwischen den beiden Grenzwerten liegen. Die Werte nähern sich um so mehr denen des $\mathrm{Ce}^{3+}$, je höher die Temperatur ist. Berechnet man nach der Mischungsregel - was sicher nur in erster Annäherung zulässig ist - aus den gefundenen Suszeptibilitäten das Verhältnis von $\mathrm{Ce}^{4+}$ zu Ce $\mathrm{Ce}^{3+}$-Ionen, so findet man: Bei $90^{\circ}$ liegen beide Ladungsstufen zu etwa gleichen Anteilen vor, bei $195^{0}$ beträgt die Zahl der $\mathrm{Ce}^{3+}$-Ionen $70 \%$, bei Zimmertemperatur $84 \%$.

Beim Praseodym scheinen auf den ersten Blick die Verhältnisse sehr viel einfacher zu sein, weil das effektive Moment unabhängig von der Temperatur ist. Jedoch liegt gerade hier ein vorläufig noch 
gänzlich unverständliches Verhalten vor; denn der für das effektive Moment gefundene Wert (3,22 Magnetonen) ist sehr viel niedriger als der für $\mathrm{Pr}^{3+}$-Ionen berechnete (3,61 Magnetonen). Eine in entsprechender Weise wie beim Cer durchgeführte Berechnung würde zu dem Schlusse führen, daß hier unabhängig von der Temperatur ein Gemisch von praktisch gleichen Mengen $\mathrm{Pr}^{3+}$ - und $\mathrm{Pr}^{4+-}$ Ionen vorliegt. Dieser Befund widerspricht allen chemischen Erfahrungen; denn Praseodym hat viel weniger Neigung, 4-wertig aufzutreten, als Cer. Es ist ferner mit dem Verlauf der Atomvolumenkurve im Widerspruch; nach den Gitterabständen kann höchstens ein ganz geringer Anteil an $\operatorname{Pr}^{4+}$-Ionen vorhanden sein. Schließlich wäre unverständlich, daß ein solches Gleichgewicht temperaturunabhängig sein sollte. Es ist zur Zeit noch nicht zu übersehen, durch welche Einflüsse das eigenartige magnetische Verhalten des Praseodyms bestimmt ist ${ }^{1}$ ).

Sehr klar liegen die Verhältnisse dagegen beim Neodym. Das effektive Moment beträgt hier 3,49 Magnetonen bei $90^{\circ}$ abs., 3,53 bei $195^{\circ}$ und 3,56 bei $293^{\circ}$ abs. Es ist also sehr wenig temperaturabhängig und entspricht recht gut dem theoretisch berechneten Wert für das $\mathrm{Nd}^{3+}$-Ion $(3,66)$. Allerdings ist hier der Schluß vom Moment auf die Ionenladung nicht eindeutig, denn für $\mathrm{Nd}^{4+}$ berechnet sich praktisch das gleiche Moment (3,62 Magnetonen). Sicher auszuschließen ist auf Grund der magnetischen Messung nur das 2-wertige Ion mit 2,8 Magnetonen. In diesem Falle ergibt sich aber die 3-Wertigkeit der in das Elektronengas eingebetteten Nd-Ionen eindeutig aus der Atomvolumenkurve.

$\mathrm{Ob}$ es richtig ist, das Samarium schon bei dieser Gruppe zu besprechen, oder ob es zu der folgenden gehört, sei dahingestellt. Die bei $90^{\circ}$ abs. gefundene Feldstärkenabhängigkeit der Suszeptibilität weist eigentlich mehr auf einen Anschluß an die Yttererden hin. Wir möchten dieses Element aber doch schon hier betrachten, da bei ihm die Verhältnisse in magnetischer Beziehung besonders verwickelt liegen. Die theoretisch berechneten Momente sowohl von $\mathrm{Sm}^{3+}$ wie von $\mathrm{Sm}^{2+}$ sind nämlich temperaturabhängig. Die zur Zeit wahrscheinlichsten $\mu_{\text {eff. }}$-Werte sind in der nachstehenden Zusammen-

1) Da wir mit der Möglichkeit rechneten, daß der Befund durch den mangelnden Reinheitsgrad unseres Praseodym-Präparates vorgetäuscht sei, haben wir noch ein $(\mathrm{Pr}+3 \mathrm{KCl})$-Gemisch untersucht, das aus einem besonders reinen Präparat hergestellt war, das wir Herrn FEIT verdanken. Das Ergebnis bestätigte unsere früheren Messungen. 
stellung mit den experimentell ermittelten $\mu_{\text {eff. }}$-Werten verglichen ${ }^{1}$ ). Man sieht, daß diese $\mu_{\text {eff. }}$-Werte zwischen den für die beiden Ionenladungen berechneten Werten liegen. Ermittelt man aus der magnetischen Suszeptibilität den Gehalt an 2-wertigen Ionen, so findet man etwas über $40 \%$ bei $90^{\circ}$ und etwas über $20^{\circ}$ bei $195^{\circ}$ und $293^{\circ}$ abs. Es liegen die Verhältnisse also ganz ähnlich wie für die 4-wertigen Ionen beim Cer, mit steigender Temperatur wächst auch hier der Gehalt an Ionen mit der normalen 3-Wertigkeit.

\begin{tabular}{r|c|c|c}
\hline \multirow{2}{*}{$T^{0}$} & \multicolumn{2}{|c|}{$\mu_{\text {theoret. }}$ für } & \multirow{2}{*}{$\mu_{\text {eff., gef. }}$} \\
\cline { 2 - 3 } & $\mathrm{Sm}^{3+}$ & $\mathrm{Sm}^{2+}$ & \\
\hline \hline 90 & $1,1_{0}$ & $2,2_{5}$ & 1,75 \\
195 & $1,3_{5}$ & $3,0_{6}$ & 1,85 \\
293 & $1,5_{5}$ & $3,4_{0}$ & 2,07
\end{tabular}

b) Europium bis Cassiopeium. Bei der Betrachtung dieser Gruppe vermittelt die Angabe der $\mu_{\text {eff. }}$-Werte wegen der ferromagnetischen Erscheinungen kein so klares Bild. Es ist hier besser, die paramagnetischen Momente nach der Formel: $\mu_{\text {para }}=2,84 \sqrt{\chi_{\text {Atom }} \cdot(T-(-))}$ zu berechnen. Diese sind in Tabelle 12 zusammengestellt. Aus dieser

Tabelle 12

\begin{tabular}{|c|c|c|c|c|c|c|}
\hline & & \multirow{2}{*}{$\Theta$-Wert } & \multirow{2}{*}{$\mu_{\text {gef. }}$} & \multicolumn{3}{|c|}{$\mu_{\text {ber. }}$ für } \\
\hline & & & & $\mathrm{Me}^{4+}$ & $\mathrm{Me}^{3+}$ & $\mathrm{Me}^{2+}$ \\
\hline $\begin{array}{l}\text { Eu. } \\
\text { Gd } \\
\text { Tb. } \\
\text { Dy } \\
\text { Ho } \\
\text { Er. } \\
\text { Tm } \\
\text { Yrb } \\
\text { Cp. }\end{array}$ & $\begin{array}{l}. \\
\therefore: \\
\therefore \\
\therefore \\
\therefore \\
\therefore \\
\therefore \\
\therefore\end{array}$ & $\begin{array}{l}+15 \\
+302 \\
+205 \\
+150 \\
+-40 \\
+10 \\
-\end{array}$ & $\begin{array}{c}8,3 \\
7,8 \\
(9,0) \\
10,9 \\
-9,5 \\
7,6 \\
0 \\
0\end{array}$ & $\begin{array}{c}\left.1,5^{2}\right) \\
\left.3,4^{2}\right) \\
7,9 \\
9,7 \\
10,6 \\
10,6 \\
9,6 \\
7,6 \\
4,5\end{array}$ & $\begin{array}{r}\left.3,4^{2}\right) \\
7,9 \\
9,7 \\
10,6 \\
10,6 \\
9,6 \\
7,6 \\
4,5 \\
0\end{array}$ & $\begin{array}{r}7,9 \\
9,7 \\
10,6 \\
10,6 \\
9,6 \\
7,6 \\
4,5 \\
0 \\
\gg 0\end{array}$ \\
\hline
\end{tabular}

Tabelle ersieht man, daß für die Elemente Gadolinium, Erbium, Thulium und Cassiopeium auf Grund der magnetischen Messungen mit Sicherheit auf die Existenz 3-wertiger Ionen geschlossen werden kann. Beim Dysprosium wären nach der magnetischen Untersuchung auch 2-wertige Ionen möglich. Hier liegt der Fall ähnlich wie beim Neodym; die Annahme 2-wertiger Ionen wird durch

1) Der ,experimentelle“ $\mu_{\text {eff. }}$.Wert für $90^{\circ}$ absolut ist durch Extrapolation auf die Feldstärke $\infty$ erhalten.

2) Für Zimmertemperatur. 
das allgemeine chemische Verhalten und die Atomvolumenkurve sicher ausgeschlossen. Ferner beweist das magnetische Verhalten, daß beim Europium und Ytterbium nicht 3-, sondern 2-wertige Ionen vorhanden sind. Beim Yb kann der Gehalt an 3-wertigen Ionen bei $90^{\circ}$ abs. höchstens $2 \%$, bei Zimmertemperatur höchstens $3 \%$ betragen. Beim Eu findet sich zwar eine kleine Abweichung gegenüber dem theoretisch für 2-wertige Ionen berechneten Wert, jedoch ist diese in Anbetracht des komplizierten magnetischen Verhaltens dieses Elementes ohne Belang.

Beim Terbium hätte man auf Grund der Atomvolumenkurve einen Wert erwartet, der nahe bei dem des 3-wertigen Ions liegt und nur einen geringen Gehalt an 4-wertigen Ionen anzeigt. Der von uns gefundene Wert könnte zu der Annahme verleiten, daß der Gehalt an 4-wertigen Ionen erheblich sei. Bei der geringen Reinheit unseres Präparates halten wir aber vorläufig einen solchen Schluß für unzulässig.

Alles in allem ergibt sich also, da $B$ das aus dem Verlaufe der Atomvolumenkurve abgeleitete Bild über den Aufbau der Metalle der seltenen Erden durch die magnetischen Messungen - mit Ausnahme von Praseodym - ausgezeichnet bestätigt wird. Darüber hinaus vermittelt aber die Betrachtung der magnetischen Momente eine wesentlich genauere Angabe der Mengenverhältnisse, in denen die verschiedenen Ionenladungen vorhanden sind. Ferner geben die ferromagnetischen Erscheinungen Hinweise, in welcher Richtung das grobe Schema: positive Ionen + Elektronengas zu verfeinern ist, um der Wirklichkeit besser zu entsprechen.

Die vorliegende Untersuchung wurde uns dadurch ermöglicht, daß uns die Herren Generaldirektor Dr. Dr.-Ing. e. h. FeIt, Prof. Dr. Jantsch, Prof. Dr. Prandtl und Prof. Dr. Tomascher durch Überlassung seltener Erden unterstützten. Wir möchten auch an dieser Stelle für dieses Entgegenkommen danken. Fräulein E. Mehnert und Herrn Brauer (Phys. Inst. der T. H. Dresden) sind wir für die röntgenspektroskopische Untersuchung unseres Europiums zu Dank verpflichtet. Ferner unterstützte uns die Deutsche Forschungsgemeinschaft durch die Überlassung von Apparaten und Geldmitteln, wofür wir auch an dieser Stelle danken.

\section{Zusammenfassung}

1. Es wurden dieMetallealler seltenen Erden (mit Ausnahme von Holmium) durch Reduktion der Erdchloride mit Alkalimetallen hergestellt. 
W. Klemm u. H. Bommer. Zur Kenntnis der Metalle der seltenen Erden 171

2. An dem so erhaltenen Gemisch von Erdmetall und Alkalichlorid wurden röntgenographische und magnetische Messungen durchgeführt.

3. Die beobachteten Gitterstrukturen entsprechen denjenigen, die nach den in den Nachbargruppen vorhandenen Gesetzmäßigkeiten zu erwarten waren.

4. Die Atomvolumenkurve zeigt ausgeprägte Maxima bei Europium und Ytterbium, flache Minima bei Cer, Praseodym und Terbium.

5. Der aus der Atomvolumenkurve zu ziehende Schluß, daß in einzelnen Metallen der seltenen Erden neben dem Elektronengas nicht nur 3-, sondern auch 2- bzw. 4-wertige Ionen vorkommen, wird durch die magnetischen Messungen bestätigt.

6. Ferromagnetisch ist unter den Metallen der seltenen Erden nur das Gadolinium. Jedoch zeigen auch die benachbarten Elemente Übergangserscheinungen vom Para- zum Ferromagnetismus.

Herrn Prof. Dr. W. Kuemr möchte ich an dieser Stelle meinen herzlichsten Dank aussprechen, daß mir Gelegenheit zur Durchführung vorliegender Arbeit in seinem Institut gegeben wurde sowie für das Interesse, das er meiner Arbeit stets entgegenbrachte. 


\section{L e b e n s l a u f}

Ich, Heinrich Bommer, wurde am 5. Oktober 1912 als Sohn des verstorbenen Schneidermeisters JoHAnnes Bommer in Röhrigshof (Werra), Hessen-Nassau, geboren. Ich bin evangelisch, besuchte von 1925 bis 1932 das Reform-Realgymnasium zu Vacha (Werra). Von Ostern 1932 bis Ostern 1936 studierte ich Chemie an derUniversität Göttingen und der Technischen Hochschule Danzig, wo ich im Mai 1936 die Diplom-Hauptprüfung ablegte. Vorliegende Dissertation führte ich im Anorganisch-chemischen Institut der.Techn. Hochschule Danzig unter Leitung von Herrn Prof. Dr. W. KLEMM aus.

Die mündliche Doktorprüfung bestand ich am 3. April 1937. 
